\title{
Methylpentanediolborane: Easy Access to New Air- and Chromatography-Stable, Highly Functionalized vinylboronates.
}

\author{
Nageswaran PraveenGanesh, Sylvain d'Hondt, Pierre Yves Chavant ${ }^{*}$
}

\section{Supplementary material}

\section{Table of contents}

Experimental details

Preparation of MPBH 1 from $\mathrm{BH}_{3}$ :DMS S3

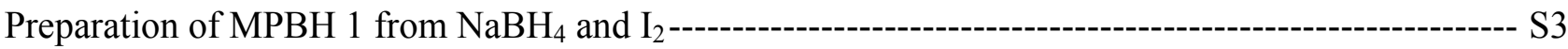

General experimental procedure for the hydroboration of alkynes by MPBH 1-------------------------- S4

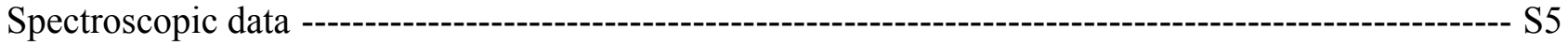

${ }^{1}$ H-NMR spectrum of 1: MPBH (4,4,6-trimethyl-1,3,2-dioxaborinane) ---------------------------------- S9

${ }^{11} \mathrm{~B}-\mathrm{NMR}$ spectrum of 1: MPBH (4,4,6-trimethyl-1,3,2-dioxaborinane)---------------------------------S10

${ }^{13} \mathrm{C}-\mathrm{NMR}$ spectrum of 1: MPBH (4,4,6-trimethyl-1,3,2-dioxaborinane)--------------------------------- S1 1

${ }^{1}$ H-NMR spectrum of 3: 4,4,6-trimethyl-2-styryl-[1,3,2]dioxaborinane----------------------------------S12

${ }^{1}$ H-NMR spectrum of 3': 4,4,5,5-tetramethyl-2-styryl-[1,3,2]dioxaborolane --------------------------- S13

${ }^{13} \mathrm{C}-\mathrm{NMR}$ spectrum of 3: 4,4,6-trimethyl-2-styryl-[1,3,2]dioxaborinane ---------------------------------S14

${ }^{11}$ B-NMR spectrum of 3: 4,4,6-trimethyl-2-styryl-[1,3,2]dioxaborinane --------------------------------- S15

${ }^{1}$ H-NMR spectrum of 4: 2-hex-1-enyl-4,4,6-trimethyl-[1,3,2]dioxaborinane-----------------------------S16

${ }^{13} \mathrm{C}-\mathrm{NMR}$ spectrum of 4: 2-hex-1-enyl-4,4,6-trimethyl-[1,3,2]dioxaborinane---------------------------S17

${ }^{1}$ H-NMR spectrum of 5: 2- cyclohexen-1-enyl-4,4,6-trimethyl-[1,3,2]dioxaborinane-------------------S18

${ }^{13} \mathrm{C}-\mathrm{NMR}$ spectrum of 5: 2- cyclohexen-1-enyl-4,4,6-trimethyl-[1,3,2]dioxaborinane---------------- S19

${ }^{1}$ H-NMR spectrum of 6: 2-(5-chloro-pent-1-enyl)-4,4,6-trimethyl-[1,3,2]dioxaborinane ------------- S20

${ }^{1} \mathrm{H}-\mathrm{NMR}$ spectrum of 6: 2-(5-chloro-pent-1-enyl)-4,4,6-trimethyl-[1,3,2]dioxaborinane -------------- S21 
${ }^{1} \mathrm{H}-\mathrm{NMR}$ spectrum of 7: 4,4,6-trimethyl-2-(2-trimethylsilanyl-vinyl)-[1,3,2]dioxaborinane -----------S22

${ }^{13} \mathrm{C}-\mathrm{NMR}$ spectrum of 7: 4,4,6-trimethyl-2-(2-trimethylsilanyl-vinyl)-[1,3,2]dioxaborinane --------- S23

${ }^{1} \mathrm{H}-\mathrm{NMR}$ spectrum of 8: 2-(3-methoxy-propenyl)-4,4,6-trimethyl-[1,3,2] dioxaborinane -------------- S24

${ }^{13} \mathrm{C}-\mathrm{NMR}$ spectrum of 8: 2-(3-methoxy-propenyl)-4,4,6-trimethyl-[1,3,2]dioxaborinane ------------- S25

${ }^{1} \mathrm{H}-\mathrm{NMR}$ spectrum of 9: 2-(3-acetoxy-propenyl)-4,4,6-trimethyl-[1,3,2]dioxaborinane --------------- S26

${ }^{13} \mathrm{C}-\mathrm{NMR}$ spectrum of 9: 2-(3-acetoxy-propenyl)-4,4,6-trimethyl-[1,3,2] dioxaborinane--------------- S27

${ }^{1} \mathrm{H}-\mathrm{NMR}$ spectrum of 11: 2-(3-tert-butoxy-propenyl)-4,4,6-trimethyl-[1,3,2]dioxaborinane---------- S28

${ }^{13} \mathrm{C}-\mathrm{NMR}$ spectrum of 11: 2-(3-tert-butoxy-propenyl)-4,4,6-trimethyl-[1,3,2]dioxaborinane--------- S29

${ }^{1} \mathrm{H}-\mathrm{NMR}$ spectrum of 12: 2-(3-chloro-propenyl)-4,4,6-trimethyl-[1,3,2]dioxaborinane --------------- S30

${ }^{13} \mathrm{C}-\mathrm{NMR}$ spectrum of 12: 2-(3-chloro-propenyl)-4,4,6-trimethyl-[1,3,2] dioxaborinane--------------- S31

${ }^{1} \mathrm{H}-\mathrm{NMR}$ spectrum of 13: 2-(3-bromo-propenyl)-4,4,6-trimethyl-[1,3,2] dioxaborinane -------------- S32

${ }^{13} \mathrm{C}-\mathrm{NMR}$ spectrum of 13: 2-(3-bromo-propenyl)-4,4,6-trimethyl-[1,3,2]dioxaborinane -------------- S33

${ }^{1} \mathrm{H}-\mathrm{NMR}$ spectrum of 14: 2-(3,3-diethoxy-propenyl)-4,4,6-trimethyl-[1,3,2]dioxaborinane ---------- S34

${ }^{13} \mathrm{C}-\mathrm{NMR}$ spectrum of 14: 2-(3,3-diethoxy-propenyl)-4,4,6-trimethyl-[1,3,2]dioxaborinane --------- S35

${ }^{1} \mathrm{H}-\mathrm{NMR}$ spectrum of 15: 3-(4,4,6-trimethyl-[1,3,2]dioxaborinan-2-yl)-acrylic acid tert-butyl ester S36

${ }^{13} \mathrm{C}-\mathrm{NMR}$ spectrum of 15: 3-(4,4,6-trimethyl-[1,3,2]dioxaborinan-2-yl)-acrylic acid tert-butyl ester S37

${ }^{1}$ H-NMR spectrum of 16: 4,4,6-trimethyl-2-phenethyl-[1,3,2]dioxaborinane --------------------------- S38

${ }^{13} \mathrm{C}-\mathrm{NMR}$ spectrum of 16: 4,4,6-trimethyl-2-phenethyl-[1,3,2]dioxaborinane --------------------------S39 


\section{Experimental Section}

All reactions were performed under nitrogen using usual Schlenk techniques, in oven-dried glassware, with magnetic stirring. Solutions of $\mathrm{BH}_{3}$ in THF $(1 \mathrm{M})$ and $\mathrm{BH}_{3}$ :DMS $(10 \mathrm{M})$ were purchased from Aldrich. DCM and 2-methyl-2,4-pentanediol were distilled from $\mathrm{CaH}_{2}$. Toluene was distilled from sodium.

Preparation of MPBH 1 from $\mathbf{B H}_{3} / \mathbf{D M S}$. Caution : Stench! $H_{2}$ evolution! A solution of 2-methyl2,4-pentanediol $(10 \mathrm{mmol}, 1.18 \mathrm{~g})$ in dry dichloromethane or toluene $(2.36 \mathrm{~mL})$ was stirred and cooled to $0{ }^{\circ} \mathrm{C}$. A solution of $\mathrm{BH}_{3}$ :DMS (11 mmol, $10 \mathrm{M}$ in methyl sulfide, Aldrich) was added over 20-30 min to control the brisk evolution of gas. The reaction was stirred for $1 \mathrm{~h}$ at $0{ }^{\circ} \mathrm{C}$ and was then warmed to 25 ${ }^{\circ} \mathrm{C}$ and stirred for an additional $1 \mathrm{hr}$ until there was no further evolution of gas. The resulting solution was checked by ${ }^{11} \mathrm{~B}$ and ${ }^{1} \mathrm{H}$ NMR; conversion of the diol was quantitative. This solution was used as such for hydroboration.

Preparation of MPBH 1 from $\mathrm{NaBH}_{4}$ and $\mathbf{I}_{2}$. Caution: Diborane is a toxic and pyrophoric gas! $\mathrm{H}_{2}$ evolution! Preparation should be carried out under an efficient fume hood! In a 100-mL double-necked round-bottomed flask fitted with a pressure-equalizing dropping funnel, an efficient magnetic stirring, and a $\mathrm{N}_{2}$ inlet, $\mathrm{NaBH}_{4}(1.14 \mathrm{~g}, 30 \mathrm{mmol})$ was suspended in $6 \mathrm{~mL}$ of bis(2-methoxyethyl) ether (diglyme). This flask was connected through a double-ended needle to a second 25 -mL cylindrical flask containing a solution of 2-methyl-2,4-pentanediol $(1.18 \mathrm{~g}, 10 \mathrm{mmol})$ in $11.8 \mathrm{~mL}$ of dry dichloromethane or toluene, placed in an ice bath. The second flask was vented through a second double-ended needle bubbling into ethanol (to trap excess diborane). The dropping funnel was loaded with a solution of $\mathrm{I}_{2}$ (15 mmol, $3.84 \mathrm{~g})$ in diglyme $(18 \mathrm{~mL})$. The $\mathrm{I}_{2}$ solution was added dropwise over $1 \mathrm{~h}$; the temperature rose gently to $40{ }^{\circ} \mathrm{C}$. The evolved gases ${ }^{1}$ were bubbled through the solution of diol 2 in the second flask.

\footnotetext{
${ }^{1}$ We observed that only $\mathrm{H}_{2}$ is evolving at the beginning of the addition, since the first step is the formation of $\mathrm{NaB}_{2} \mathrm{H}_{7}$ : Brown, H. C.; Tierney, P. A. J. Am. Chem. Soc. 1958, 80, 1552.
} 
All along the process, a small stream of $\mathrm{N}_{2}$ (2-5 bubbles per second) was applied through the whole apparatus to ensure complete transfer of the diborane. At the end of the addition of $\mathrm{I}_{2}$, the temperature of the second flask was brought to $20^{\circ} \mathrm{C}$, and the stream of $\mathrm{N}_{2}$ was continued for $1 \mathrm{~h}$; this step allowed the elimination of any excess diborane in the solution of MPBH 1. The solution was transferred to a volumetric flask and diluted to $20 \mathrm{~mL} .{ }^{11} \mathrm{~B}$ and ${ }^{1} \mathrm{H}$ NMR indicated that the conversion of the diol was quantitative. Thus, the solution was considered $0.50 \mathrm{M}$ in MPBH 1 . The solution could be kept at $4{ }^{\circ} \mathrm{C}$ for several months without change. Storage at room temperature was deleterious.

\section{General Experimental Procedure for the Hydroboration of Alkynes by MPBH 1:}

A 5-mL round-bottom flask equipped with a side arm and magnetic stirring was charged with $\mathrm{HZrCp}_{2} \mathrm{Cl}(0.05 \mathrm{mmol}, 13 \mathrm{mg})$ and dry dichloromethane or toluene $(1 \mathrm{~mL})$ under a $\mathrm{N}_{2}$ atmosphere and cooled to $0^{\circ} \mathrm{C}$. Alkyne $(1.0 \mathrm{mmol})$ was then added dropwise, the mixture then was stirred for $2 \mathrm{~min}$, and MPBH $1(1.1 \mathrm{mmol})$ was introduced dropwise via a syringe. Stirring was continued for $16 \mathrm{~h}$ at $20{ }^{\circ} \mathrm{C}$, at which point $\mathrm{GC}$ analysis of a reaction aliquot showed completion of reaction. Isolation of the product was achieved by removing volatile matter under reduced pressure followed by flash column chromatography ( $3 \%$ ether in hexane) to provide the desired product as a clear oil.

All products were found to be $>95 \%$ purity by gas chromatography (FID).

The above procedure was also applied for the hydroboration of styrene by changing the catalyst to $\left(\mathrm{PPh}_{3}\right)_{3} \mathrm{RhCl}(0.01 \mathrm{mmol}, 9 \mathrm{mg})$.

Spectroscopic Data In the $75.5 \mathrm{MHz}{ }^{13} \mathrm{C}$-NMR spectra, $C$ atoms that are directly bonded to boron, marked (br) were approximately $250 \mathrm{~Hz}$ wide, and can easily remain undetected.

1: MPBH (4,4,6-Trimethyl-1,3,2-dioxaborinane); ${ }^{1} \mathrm{H}$ NMR $\left(300 \mathrm{MHz}, C D C l_{3}\right) \delta$ ppm 4.16 (dqd, $J$ $=11.8,6.2,3.0 \mathrm{~Hz}, 1 \mathrm{H}), 1.76(\mathrm{ddd}, J=13.9,3.0,1.7 \mathrm{~Hz}, 1 \mathrm{H}), 1.50(\mathrm{dd}, J=13.9,11.8 \mathrm{~Hz}, 1 \mathrm{H}), 1.27(\mathrm{~s}$, $3 \mathrm{H}), 1.26(\mathrm{~s}, 3 \mathrm{H}), 1.23(\mathrm{~d}, J=6.21 \mathrm{~Hz}, 3 \mathrm{H}) .{ }^{13} \mathrm{C} \mathrm{NMR}\left(75.5 \mathrm{MHz}, C D C l_{3}\right) \delta \mathrm{ppm} 70.8,64.6,46.1,31.0$, 28.1, 22.9. ${ }^{11} \mathrm{~B}$ NMR $\left(96 \mathrm{MHz}, C D C l\right.$ ) $\delta$ ppm $25.2(\mathrm{~d}, J=170 \mathrm{~Hz}) . \mathrm{IR}$ (neat, $\left.\mathrm{cm}^{-1}\right): 2551,2498$. 
3: 4,4,6-Trimethyl-2-styryl-[1,3,2] dioxaborinane; ${ }^{1} \mathrm{H}$ NMR $\left(300 \mathrm{MHz}, C D C l_{3}\right) \delta$ ppm 7.5-7.4 (m, 2H), 7.35-7.2 (m, 4H), $6.11(\mathrm{~d}, J=18.2 \mathrm{~Hz}, 1 \mathrm{H}), 4.26(\mathrm{dqd}, J=11.7,6.2,3.0 \mathrm{~Hz}, 1 \mathrm{H}), 1.80(\mathrm{dd}, J=$ 13.8, 3.0 Hz, 1H), $1.53(\mathrm{dd}, J=13.8,11.7 \mathrm{~Hz}, 1 \mathrm{H}), 1.33(\mathrm{~s}, 3 \mathrm{H}), 1.32(\mathrm{~s}, 3 \mathrm{H}), 1.30(\mathrm{~d}, J=6.2 \mathrm{~Hz}$, $3 \mathrm{H}) .{ }^{13} \mathrm{C}$ NMR $\left(75.5 \mathrm{MHz}, C D C l_{3}\right) \delta \mathrm{ppm} 146.4,137.9,128.3,128.2,126.8,121$ (br), 70.7, 64.7, 45.9, 31.2, 28.1, 23.1. ${ }^{11} \mathrm{~B}$ NMR (96 MHz, $\left.C D C l 3\right) \delta 25.8$ ppm. IR $\left(\mathrm{cm}^{-1}\right): 3023,2974,1624,1577,1213$,

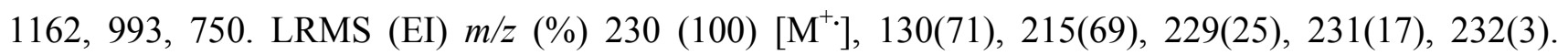
HRMS EI $m / z$ calculated for $\mathrm{C}_{14} \mathrm{H}_{19} \mathrm{BO}_{2}{ }^{+} 230.1473$; found 230.1478 .

4: 2-Hex-1-enyl-4,4,6-trimethyl-[1,3,2] dioxaborinane; ${ }^{1} \mathrm{H}$ NMR $\left(300 \mathrm{MHz}, C D C l_{3}\right) \delta \mathrm{ppm} 6.52(\mathrm{dt}$, $J=17.7,6.4 \mathrm{~Hz}, 1 \mathrm{H}), 5.34(\mathrm{dt}, J=17.7,1.6 \mathrm{~Hz}, 1 \mathrm{H}), 4.20(\mathrm{dqd}, J=11.8,6.2,2.9 \mathrm{~Hz}, 1 \mathrm{H}), 2.12(\mathrm{~m}$, 2H), $1.77(\mathrm{dd}, J=13.9,2.9 \mathrm{~Hz}, 1 \mathrm{H}), 1.49(\mathrm{dd}, J=13.9,11.8 \mathrm{~Hz}, 1 \mathrm{H}), 1.43-1.30(\mathrm{~m}, 4 \mathrm{H}), 1.29(\mathrm{~s}, 6 \mathrm{H})$,

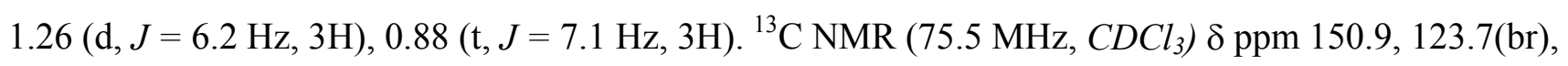
70.3, 64.4, 45.9, 35.0, 31.2, 30.5, 28.0, 23.1, 22.2, 13.8. ${ }^{11} \mathrm{~B} \mathrm{NMR}\left(96 \mathrm{MHz}, C D C l_{3}\right) \delta 25.6 \mathrm{ppm} . \mathrm{IR}$ $\left(\mathrm{cm}^{-1}\right): 2973,1639,1388,1164,998,768$. LRMS (DCI NH$/$ Isobutane) $m / z(\%) 228(100)\left[\mathrm{M}+\mathrm{NH}_{4}^{+}\right]$. HRMS (DCI NH 3$) ~ m / z$ calculated for $\mathrm{C}_{12} \mathrm{H}_{27} \mathrm{BNO}_{2}{ }^{+} 228.2129$; found 228.2135

5: 2- cyclohexen-1-enyl-4,4,6-trimethyl-[1,3,2]dioxaborinane; ${ }^{1} \mathrm{H}$ NMR $\left(300 \mathrm{MHz}, \mathrm{CDCl}_{3}\right) \delta \mathrm{ppm}$ $6.91(\mathrm{~d}, J=17.6 \mathrm{~Hz}, 1 \mathrm{H}), 5.88$ (br. t, $J=3.2 \mathrm{~Hz}, 1 \mathrm{H}), 5.34(\mathrm{~d}, J=17.6 \mathrm{~Hz}, 1 \mathrm{H}), 4.20$ (dqd, $J=11.8$, 6.2, $3.0 \mathrm{~Hz}, 1 \mathrm{H}), 2.18-2.04(\mathrm{~m}, 2 \mathrm{H}), 1.76(\mathrm{dd}, J=13.9,3.0 \mathrm{~Hz}, 1 \mathrm{H}), 1.68-1.53(\mathrm{~m}, 4 \mathrm{H}), 1.47(\mathrm{dd}, J=$ 13.9, $11.8 \mathrm{~Hz}, 1 \mathrm{H}), 1.30-1.27(\mathrm{~m}, 8 \mathrm{H}), 1.25(\mathrm{~d}, J=6.2 \mathrm{~Hz}, 3 \mathrm{H}) .{ }^{13} \mathrm{C} \mathrm{NMR}\left(75.5 \mathrm{MHz}, C D C l_{3}\right) \delta \mathrm{ppm}$ 150.1, 137.1(br), 132.4, 117.4, 70.4, 64.5, 45.9, 31.1, 28.0, 25.9, 23.9, 23.1, 22.4, 22.3. ${ }^{11} \mathrm{~B}$ NMR (96 $\left.\mathrm{MHz}, \mathrm{CDCl}_{3}\right) \delta 26.0$ ppm. IR $\left(\mathrm{cm}^{-1}\right): 3018,2974,2929,1634,1607,1300,1162,997,795,771 . \mathrm{LRMS}$ (DCI NH$/$ Isobutane) $m / z(\%) 235(100)\left[\mathrm{M}+\mathrm{H}^{+}\right], 247(40), 248(23), 250(34), 251(4) . \mathrm{HRMS}\left(\mathrm{DCI} \mathrm{NH}_{3}\right)$ $m / z$ calculated for $\mathrm{C}_{14} \mathrm{H}_{24} \mathrm{BO}_{2} 235.1864$; found 235.1861

6: 2-(5-Chloro-pent-1-enyl)-4,4,6-trimethyl-[1,3,2]dioxaborinane; ${ }^{1} \mathrm{H}$ NMR $\left(200 \mathrm{MHz}, C D C l_{3}\right) \delta$ ppm $6.42(\mathrm{dt}, J=17.7,6.4 \mathrm{~Hz}, 1 \mathrm{H}), 5.33(\mathrm{dt}, J=17.7,1.5 \mathrm{~Hz}, 1 \mathrm{H}), 4.16(\mathrm{dqd}, J=11.7,6.2,3.0 \mathrm{~Hz}$, 
$1 \mathrm{H}), 3.48(\mathrm{t}, J=6.7 \mathrm{~Hz}, 2 \mathrm{H}), 2.29-2.13(\mathrm{tt}, J=8.1,6.7 \mathrm{~Hz}, 2 \mathrm{H}), 1.85(\mathrm{q}, J=8.1 \mathrm{~Hz}, 1 \mathrm{H}), 1.73(\mathrm{dd}, J=$ 13.9, 3.0 Hz, 1H), $1.44(\mathrm{dd}, J=13.9,11.7 \mathrm{~Hz}, 1 \mathrm{H}), 1.24(\mathrm{~s}, 6 \mathrm{H}), 1.21(\mathrm{~d}, J=6.2 \mathrm{~Hz}, 3 \mathrm{H}) .{ }^{13} \mathrm{C} \mathrm{NMR}$ $\left(75.5 \mathrm{MHz}, \mathrm{CDCl}_{3}\right) \delta \mathrm{ppm} 148.3,125.2(\mathrm{br}), 70.5,64.5,45.9,44.3,32.3,31.2(\mathrm{x} 2), 28.0,23.0 .{ }^{11} \mathrm{~B}$ NMR $\left(96 \mathrm{MHz}, C D C l_{3}\right) \delta 25.5$ ppm. IR $\left(\mathrm{cm}^{-1}\right): 2970,2933,1641,1392,1164,995,771$. LRMS (DCI $\mathrm{NH}_{3} /$ Isobutane) $m / z(\%) 248(100)\left[\mathrm{M}+\mathrm{NH}_{4}{ }^{+}\right], 234(11), 236(7)$. HRMS (DCI $\left.\mathrm{NH}_{3}\right) \mathrm{m} / z$ calculated for $\mathrm{C}_{11} \mathrm{H}_{24} \mathrm{BClNO}_{2} 248.1583$; found 248.1586

7: 4,4,6-Trimethyl-2-(2-trimethylsilanylvinyl)-[1,3,2]dioxaborinane; ${ }^{1} \mathrm{H} \quad \mathrm{NMR} \quad(300 \mathrm{MHz}$, $\left.C D C l_{3}\right) \delta$ ppm $7.01(\mathrm{~d}, J=21.6 \mathrm{~Hz}, 1 \mathrm{H}), 6.18(\mathrm{~d}, J=21.6 \mathrm{~Hz}, 1 \mathrm{H}), 4.24(\mathrm{dqd}, J=11.7,6.2,3.0 \mathrm{~Hz}$, 1H), 1.79 (dd, $J=13.8,3.0 \mathrm{~Hz}, 1 \mathrm{H}), 1.51(\mathrm{dd}, J=13.8,11.7 \mathrm{~Hz}, 1 \mathrm{H}), 1.32(\mathrm{~s}, 3 \mathrm{H}), 1.31(\mathrm{~s}, 3 \mathrm{H}), 1.29$ $(\mathrm{d}, J=6.2 \mathrm{~Hz}, 3 \mathrm{H}), 0.07$ (s, 9H). ${ }^{13} \mathrm{C}$ NMR $\left(75.5 \mathrm{MHz}, C D C l_{3}\right) \delta \mathrm{ppm}: 153.3,142(\mathrm{br}), 70.7,64.7,45.9$, 31.1, 28.0, 26.8, 23.1, -1.6. ${ }^{11} \mathrm{~B}$ NMR (96 MHz, $\left.C D C l_{3}\right) \delta 24.6$ ppm. IR $\left(\mathrm{cm}^{-1}\right): 2970,1592,1418,1389$, 1018, 862, 1166, 837, 744. LRMS (DCI NH $3 /$ Isobutane) $m / z(\%) 228(100)\left[\mathrm{M}+\mathrm{NH}_{4}{ }^{+}\right], 209(9), 243(36)$, 245(12). HRMS (DCI NH 3$) ~ m / z$ calculated for $\mathrm{C}_{11} \mathrm{H}_{27} \mathrm{BNO}_{2} \mathrm{Si}=244.1899$; found 244.1909

8: 2-(3-Methoxy-propenyl)-4,4,6-trimethyl-[1,3,2]dioxaborinane; ${ }^{1} \mathrm{H}$ NMR $\left(200 \mathrm{MHz}, \mathrm{CDCl}_{3}\right) \delta$ ppm $6.46(\mathrm{dt}, J=17.9,5.1,1 \mathrm{H}), 5.52(\mathrm{dt}, J=17.9,1.3 \mathrm{~Hz}, 1 \mathrm{H}), 4.16(\mathrm{dqd}, J=11.6,6.3,3.0 \mathrm{~Hz}, 1 \mathrm{H})$, $3.91(\mathrm{dd}, J=5.1,1.3 \mathrm{~Hz}, 2 \mathrm{H}), 3.28(\mathrm{~s}, 3 \mathrm{H}), 1.73(\mathrm{dd}, J=13.8,3.0 \mathrm{~Hz}, 1 \mathrm{H}), 1.43(\mathrm{dd}, J=13.8,11.6 \mathrm{~Hz}$, $1 \mathrm{H}), 1.24(\mathrm{~s}, 6 \mathrm{H}), 1.20(\mathrm{~d}, J=6.3 \mathrm{~Hz}, 3 \mathrm{H}) .{ }^{13} \mathrm{C} \mathrm{NMR}\left(75.5 \mathrm{MHz}, C D C l_{3}\right) \delta \mathrm{ppm} 145.5,126(\mathrm{br}), 74.4$, 70.6, 64.6, 58.0, 45.9, 31.1, 28.0, 23.0. ${ }^{11} \mathrm{~B}$ NMR (96 MHz, $\left.C D C l_{3}\right) \delta 25.5$ ppm. IR $\left(\mathrm{cm}^{-1}\right): 2974,1644$, 1392, 1120, 1001, 768, 620. LRMS (DCI NH$/$ Isobutane) $m / z(\%) 216(100)\left[\mathrm{M}+\mathrm{NH}_{4}{ }^{+}\right], 184(26), 199$ (20), 215(36), 217(14). HRMS (DCI NH 3$) ~ m / z$ calculated for $\mathrm{C}_{10} \mathrm{H}_{23} \mathrm{BNO}_{3}{ }^{+} 216.1766$, found 216.1708

9: 2-(3-Acetoxy-propenyl)-4,4,6-trimethyl-[1,3,2]dioxaborinane; ${ }^{1} \mathrm{H}$ NMR $\left(200 \mathrm{MHz}, \mathrm{CDCl}_{3}\right) \delta$ ppm $6.44(\mathrm{td}, J=17.8,4.9 \mathrm{~Hz}, 1 \mathrm{H}), 5.52(\mathrm{td}, J=17.8,1.7 \mathrm{~Hz}, 1 \mathrm{H}), 4.55(\mathrm{dd}, J=4.9,1.7 \mathrm{~Hz}, 2 \mathrm{H}), 4.15$ $(\mathrm{dqd}, J=11.6,6.2,3.1 \mathrm{~Hz}, 1 \mathrm{H}), 2.01(\mathrm{~s}, 3 \mathrm{H}), 1.73(\mathrm{dd}, J=13.9,3.1 \mathrm{~Hz}, 1 \mathrm{H}), 1.42(\mathrm{dd}, J=13.9,11.6$ $\mathrm{Hz}, 1 \mathrm{H}), 1.22(\mathrm{~s}, 6 \mathrm{H}), 1.19(\mathrm{~d}, J=6.2 \mathrm{~Hz}, 3 \mathrm{H}) .{ }^{13} \mathrm{C} \mathrm{NMR}\left(75.5 \mathrm{MHz}, C D C l_{3}\right) \delta \mathrm{ppm} \mathrm{170.4,} \mathrm{142.3,}$ 
125(br), 70.7, 65.6, 64.6, 45.8, 31.0, 27.9, 22.9, 20.7. ${ }^{11} \mathrm{~B}$ NMR $\left(96 \mathrm{MHz}, C D C l_{3}\right) \delta 25.6$ ppm. IR $\left(\mathrm{cm}^{-}\right.$ $\left.{ }^{1}\right): 3015,2975,2935,1744,1163,993,768$. LRMS (DCI NH $3 /$ Isobutane) $m / z(\%) 227(100)\left[\mathrm{M}+\mathrm{H}^{+}\right]$, 244(52). HRMS (DCI NH 3$) ~ m / z$ calculated for $\mathrm{C}_{11} \mathrm{H}_{23} \mathrm{BNO}_{4}{ }^{+} 244.1715$; found 244.1708

11: 2-(3-tert-Butoxy-propenyl)-4,4,6-trimethyl-[1,3,2]dioxaborinane; ${ }^{1} \mathrm{H} \quad \mathrm{NMR} \quad(300 \mathrm{MHz}$, $\left.C D C l_{3}\right) \delta \operatorname{ppm} 6.48(\mathrm{dt}, J=17.8,4.9 \mathrm{~Hz}, 1 \mathrm{H}), 5.52(\mathrm{dt}, J=17.8,1.2 \mathrm{~Hz}, 1 \mathrm{H}), 4.12(\mathrm{dqd}, J=12.1,6.2$, $2.9 \mathrm{~Hz}, 1 \mathrm{H}), 3.88(\mathrm{dd}, J=4.9,1.2 \mathrm{~Hz}, 2 \mathrm{H}), 1.69(\mathrm{dd}, J=13.9,2.9 \mathrm{~Hz}, 1 \mathrm{H}), 1.39(\mathrm{dd}, J=13.9,12.1 \mathrm{~Hz}$, $1 \mathrm{H}), 1.20(\mathrm{~s}, 6 \mathrm{H}), 1.17(\mathrm{~d}, J=6.2 \mathrm{~Hz}, 3 \mathrm{H}), 1.12(\mathrm{~s}, 9 \mathrm{H}) .{ }^{13} \mathrm{C} \mathrm{NMR}\left(75.5 \mathrm{MHz}, C D C l_{3}\right) \delta \mathrm{ppm} \mathrm{147.3,}$ 123.4(br), 72.8, 70.4, 64.4, 63.9, 45.9, 31.1, 27.9, 27.4 (x3), 23.0. ${ }^{11} \mathrm{~B}$ NMR $\left(96 \mathrm{MHz}, C D C l_{3}\right) \delta 25.4$ ppm. IR $\left(\mathrm{cm}^{-1}\right): 1644,1391,997,768,620$. LRMS (DCI NH $3 /$ Isobutane) $m / z(\%) 258(100)\left[\mathrm{M}^{2} \mathrm{NH}_{4}^{+}\right]$, 202(39), 256(11), 257(21), 259(6). HRMS (ESI) Calcd for $\mathrm{C}_{13} \mathrm{H}_{25} \mathrm{O}_{3} \mathrm{BNa}^{+}$263.17890(100), 262.18253(25), 264.18226(14) Found 263.17891(100), 262.18258(25), 264.18213(14).

12: 2-(3-Chloropropenyl)-4,4,6-trimethyl-[1,3,2]dioxaborinane; ${ }^{1} \mathrm{H}$ NMR $\left(200 \mathrm{MHz}, C D C l_{3}\right) \delta$ ppm $6.52(\mathrm{dt}, J=17.4,6.3 \mathrm{~Hz}, 1 \mathrm{H}), 5.62(\mathrm{dt}, J=17.4,1.4 \mathrm{~Hz}, 1 \mathrm{H}), 4.22(\mathrm{dqd}, J=11.5,6.2,3.0 \mathrm{~Hz}$, $1 \mathrm{H}), 4.07(\mathrm{dd}, J=6.3,1.4 \mathrm{~Hz}, 2 \mathrm{H}), 1.80(\mathrm{dd}, J=13.9,3.0 \mathrm{~Hz}, 1 \mathrm{H}), 1.49(\mathrm{dd}, J=13.9,11.5 \mathrm{~Hz}, 1 \mathrm{H})$,

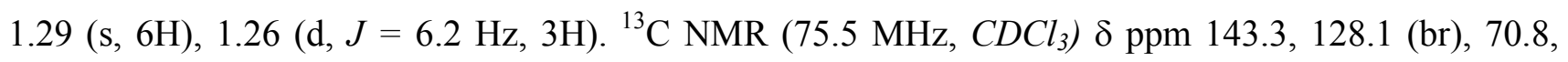
64.7, 46.2, 45.8, 31.0, 27.9, 22.9. ${ }^{11} \mathrm{~B}$ NMR $\left(96 \mathrm{MHz}, C D C l_{3}\right) \delta 25.3$ ppm. IR ( $\left.\mathrm{cm}^{-1}\right): 2974,1641,1421$, $1162,993,767$. We could not obtain any coherent mass spectrum for this compound.

13: 2-(3-Bromopropenyl)-4,4,6-trimethyl-[1,3,2]dioxaborinane; ${ }^{1} \mathrm{H}$ NMR $\left(300 \mathrm{MHz}, C D C l_{3}\right) \delta$ ppm $6.50(\mathrm{td}, J=17.3,7.2 \mathrm{~Hz}, 1 \mathrm{H}), 5.51(\mathrm{td}, J=17.3,1.1 \mathrm{~Hz}, 1 \mathrm{H}), 4.13(\mathrm{dqd}, J=11.8,6.2,3.0 \mathrm{~Hz}$, $1 \mathrm{H}), 3.88(\mathrm{dd}, J=7.16,1.1 \mathrm{~Hz}, 2 \mathrm{H}), 1.71(\mathrm{dd}, J=13.9,3.0 \mathrm{~Hz}, 1 \mathrm{H}), 1.41(\mathrm{dd}, J=13.9,11.8 \mathrm{~Hz}, 1 \mathrm{H})$, $1.21(\mathrm{~s}, 6 \mathrm{H}), 1.18(\mathrm{~d}, J=6.2 \mathrm{~Hz}, 3 \mathrm{H}) .{ }^{13} \mathrm{C} \mathrm{NMR}\left(75.5 \mathrm{MHz}, C D C l_{3}\right) \delta \mathrm{ppm} 143.5,128.6(\mathrm{br}), 70.9,64.7$, 45.8, 34.1, 31.1, 28.0, 23.0. ${ }^{11} \mathrm{~B}$ NMR $\left(96 \mathrm{MHz}, C D C l_{3}\right) \delta 25.2 \mathrm{ppm} . \mathrm{IR}\left(\mathrm{cm}^{-1}\right): 2973,2933,1634,1425$, $1230,1196,1162,993,771$. We could not obtain any coherent mass spectrum for this compound. 
14: 2-(3,3-Diethoxypropenyl)-4,4,6-trimethyl-[1,3,2] dioxaborinane; ${ }^{1} \mathrm{H}$ NMR $\left(300 \mathrm{MHz}, \mathrm{CDCl}_{3}\right) \delta$ ppm $6.34(\mathrm{dd}, J=18.0,5.0 \mathrm{~Hz}, 1 \mathrm{H}), 5.60(\mathrm{dd}, J=18.0,1.2 \mathrm{~Hz}, 1 \mathrm{H}), 4.78(\mathrm{dd}, J=5.0,1.2 \mathrm{~Hz}, 1 \mathrm{H})$, $4.13(\mathrm{dqd}, J=11.7,6.2,3.0 \mathrm{~Hz}, 1 \mathrm{H}), 3.57(\mathrm{~m}, 2 \mathrm{H}), 3.44(\mathrm{dq}, J=9.5,7.1 \mathrm{~Hz}, 2 \mathrm{H}), 1.71(\mathrm{dd}, J=13.8$, $3.0 \mathrm{~Hz}, 1 \mathrm{H}), 1.40(\mathrm{dd}, J=13.8,11.7 \mathrm{~Hz}, 1 \mathrm{H}), 1.21(\mathrm{~s}, 6 \mathrm{H}), 1.18(\mathrm{~d}, J=6.2 \mathrm{~Hz}, 3 \mathrm{H}), 1.13(\mathrm{t}, J=7.1 \mathrm{~Hz}$, $6 \mathrm{H}) .{ }^{13} \mathrm{C}$ NMR $\left(75.5 \mathrm{MHz}, \mathrm{CDCl}_{3}\right) \delta \mathrm{ppm} 145.2,126.9(\mathrm{br}), 102.4,70.7,64.6,61.0(\mathrm{x} 2), 45.9,31.1,28.0$, 23.0, 15.1(x2). ${ }^{11} \mathrm{~B}$ NMR (96 MHz, $\left.C D C l_{3}\right) \delta 25.5$ ppm. IR $\left(\mathrm{cm}^{-1}\right): 2971,2933,1648,1421,1054,1000$, 896, 767. We could not obtain any coherent mass spectrum for this compound.

15: 3-(4,4,6-Trimethyl-[1,3,2]dioxaborinan-2-yl)-acrylic Acid tert-Butyl Ester; ${ }^{1} \mathrm{H}$ NMR (300 $\left.\mathrm{MHz}, C D C l_{3}\right) \delta \mathrm{ppm} 6.61(\mathrm{~d}, J=18.0 \mathrm{~Hz}, 1 \mathrm{H}), 6.46(\mathrm{~d}, J=18.0 \mathrm{~Hz}, 1 \mathrm{H}), 4.24(\mathrm{dqd}, J=12.3,6.2,3.0$ $\mathrm{Hz}, 1 \mathrm{H}), 1.82(\mathrm{dd}, J=14.0,3.0 \mathrm{~Hz}, 1 \mathrm{H}), 1.47(\mathrm{~s}, 9 \mathrm{H}), 1.46(\mathrm{~m}, 1 \mathrm{H}), 1.30(\mathrm{~s}, 6 \mathrm{H}), 1.27(\mathrm{~d}, J=6.19 \mathrm{~Hz}$ $3 \mathrm{H}) .{ }^{13} \mathrm{C}$ NMR $\left(75.5 \mathrm{MHz}, C D C l_{3}\right) \delta \mathrm{ppm} \mathrm{165.5,} \mathrm{138(br),} \mathrm{137.8,} \mathrm{79.8,} \mathrm{71.0,} \mathrm{64.9,} \mathrm{45.7,} \mathrm{30.9,} \mathrm{27.9(3x),}$ 22.8. ${ }^{11} \mathrm{~B}$ NMR $\left(96 \mathrm{MHz}, C D C l_{3}\right) \delta 25.4 \mathrm{ppm} . \mathrm{IR}\left(\mathrm{cm}^{-1}\right): 3046,2971,2933,1714,1623,1432,1150$, 1003, 765. LRMS (DCI NH$/$ Isobutane) $m / z(\%)$ 272(57) $\left[\mathrm{M}+\mathrm{NH}_{4}{ }^{+}\right], 215(31), 216(100), 217(24)$, 271(35), 273(7). HRMS (ESI) Calcd. for $\mathrm{C}_{26} \mathrm{H}_{46} \mathrm{O}_{8} \mathrm{~B}_{2} \mathrm{Na}^{+}$531.32710(100), 530.33073(50), 532.33054(29) Found 531.32705(100), 530.33071(41), 532.32991(42).

16: 4,4,6-Trimethyl-2-phenethyl-[1,3,2] dioxaborinane; ${ }^{1} \mathrm{H}$ NMR $\left(300 \mathrm{MHz}, C D C l_{3}\right) \delta$ ppm 7.27$7.08(\mathrm{~m}, 5 \mathrm{H}), 4.13(\mathrm{dqd}, J=11.7,6.2,3.0 \mathrm{~Hz}, 1 \mathrm{H}), 1.71(\mathrm{dd}, J=13.9,3.0 \mathrm{~Hz}, 1 \mathrm{H}), 2.72-2.65(\mathrm{~m}, 2 \mathrm{H})$, $1.39(\mathrm{dd}, J=13.9,11.7 \mathrm{~Hz}, 1 \mathrm{H}), 1.24(\mathrm{~s}, 3 \mathrm{H}), 1.23(\mathrm{~s}, 3 \mathrm{H}), 1.21(\mathrm{~d}, J=6.2 \mathrm{~Hz}, 3 \mathrm{H}), 1.02(\mathrm{~m}, 2 \mathrm{H}) .{ }^{13} \mathrm{C}$ NMR $\left(75.5 \mathrm{MHz}, \mathrm{CDCl}_{3}\right) \delta \mathrm{ppm} 145.1,128.0,127.9,125.1,70.4,64.4,45.9,31.1,30.2,28.0,23.1$, 17(br). ${ }^{11}$ B NMR (96 MHz, $C D C l$ ) $\delta 29.7$ ppm. IR $\left(\mathrm{cm}^{-1}\right): 3024,2974,2933,1389,1182,793,771,751$. LRMS (DCI $\mathrm{NH}_{3} /$ Isobutane) $m / z(\%)$ 250(100) $\left[\mathrm{M}+\mathrm{NH}_{4}{ }^{+}\right.$]. HRMS (DCI $\mathrm{NH}_{3}$ ) $\mathrm{m} / z$ calculated for $\mathrm{C}_{14} \mathrm{H}_{25} \mathrm{BNO}_{2}^{+}=250.1973$; found 250.1966. 

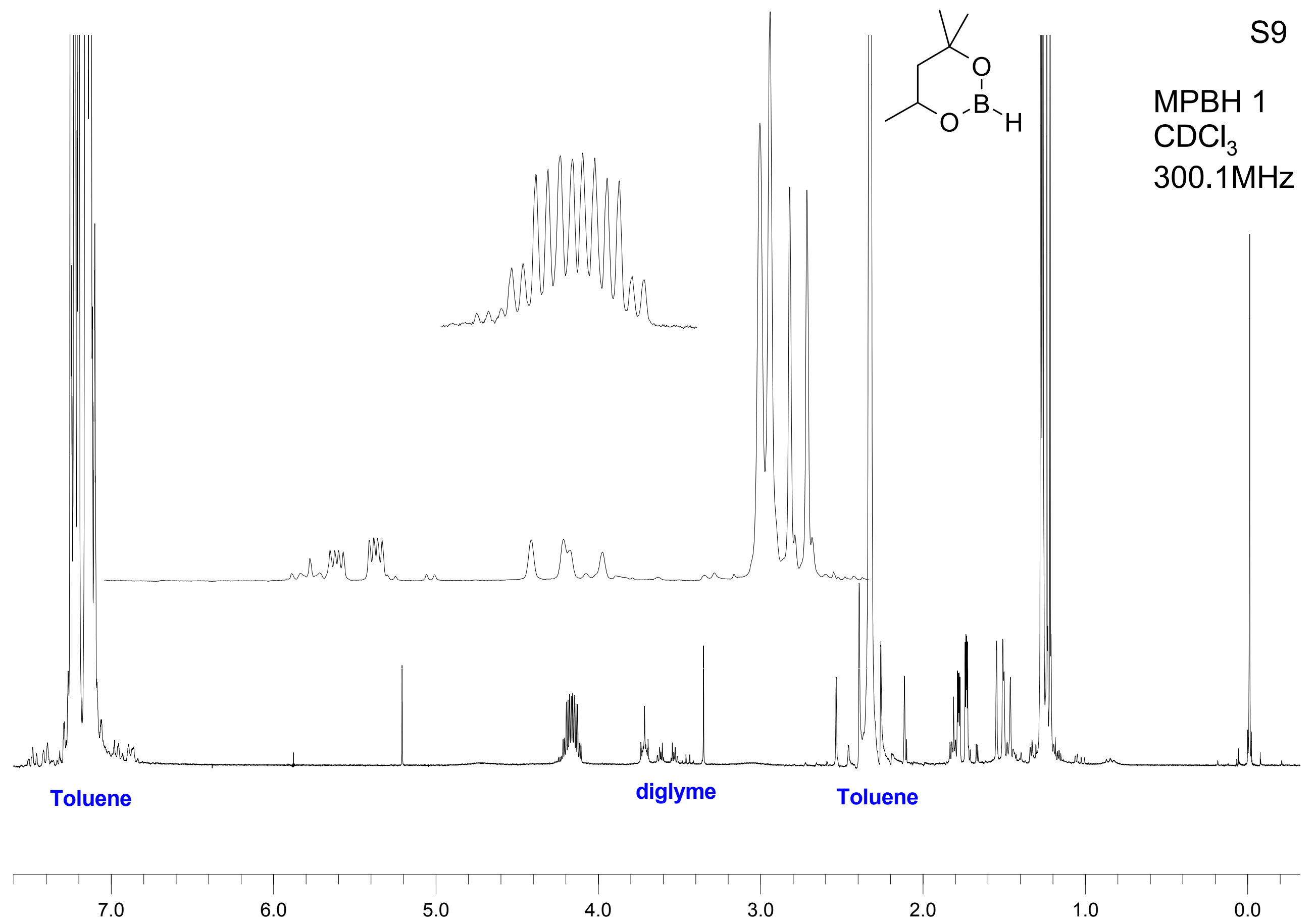

ppm (t1) 


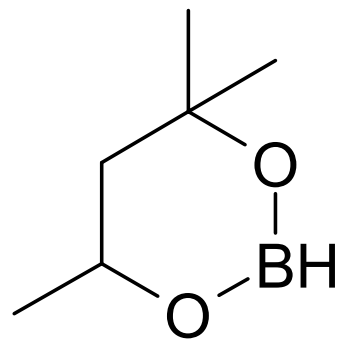

\section{MPBH 1 \\ ${ }^{11} \mathrm{~B} N M R$ $\mathrm{CDCl}_{3}$ $96 \mathrm{MHz}$ \\ $\delta$ ppm 25.17 \\ $(d, J=170.2 \mathrm{~Hz})$}




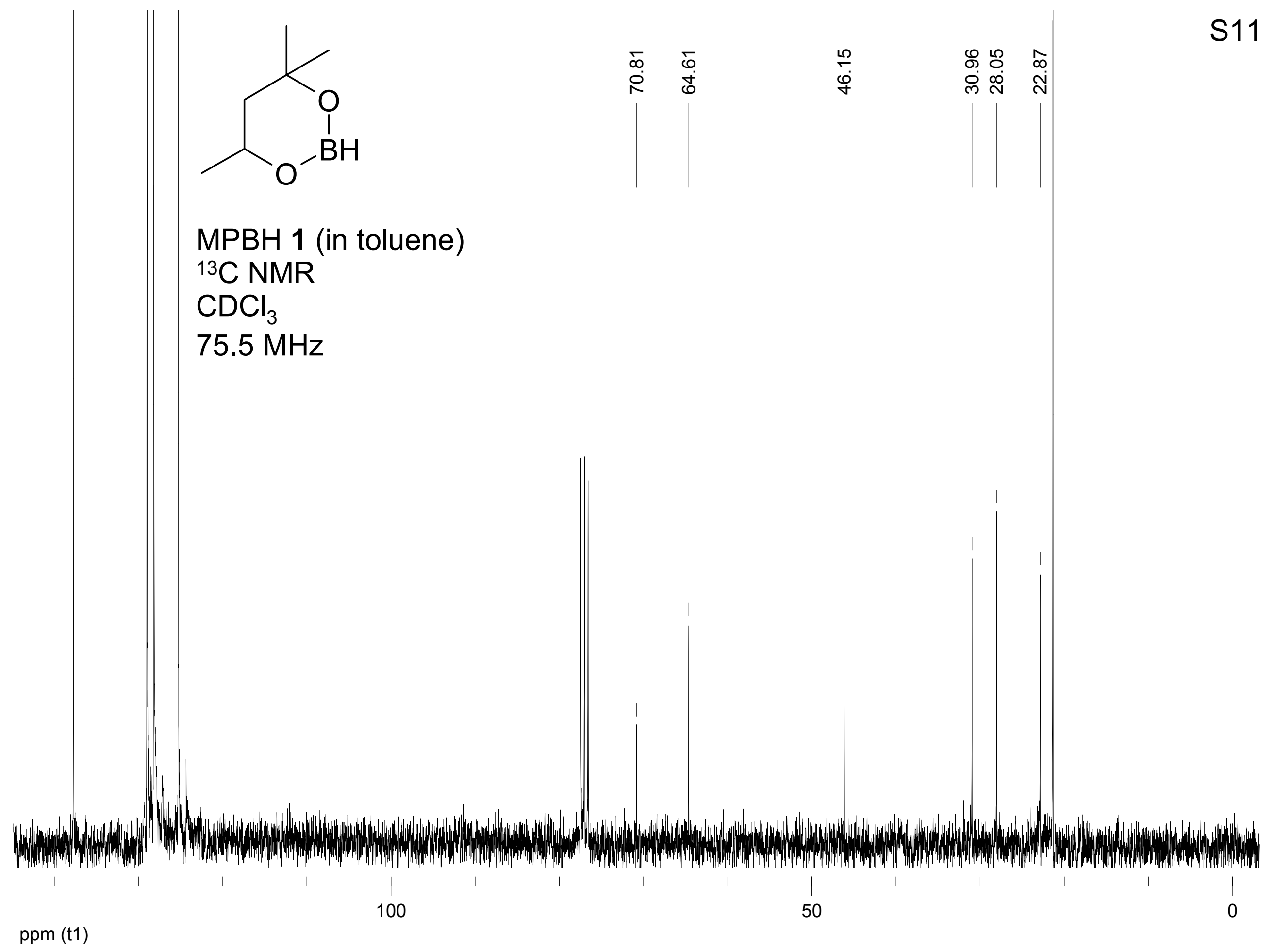



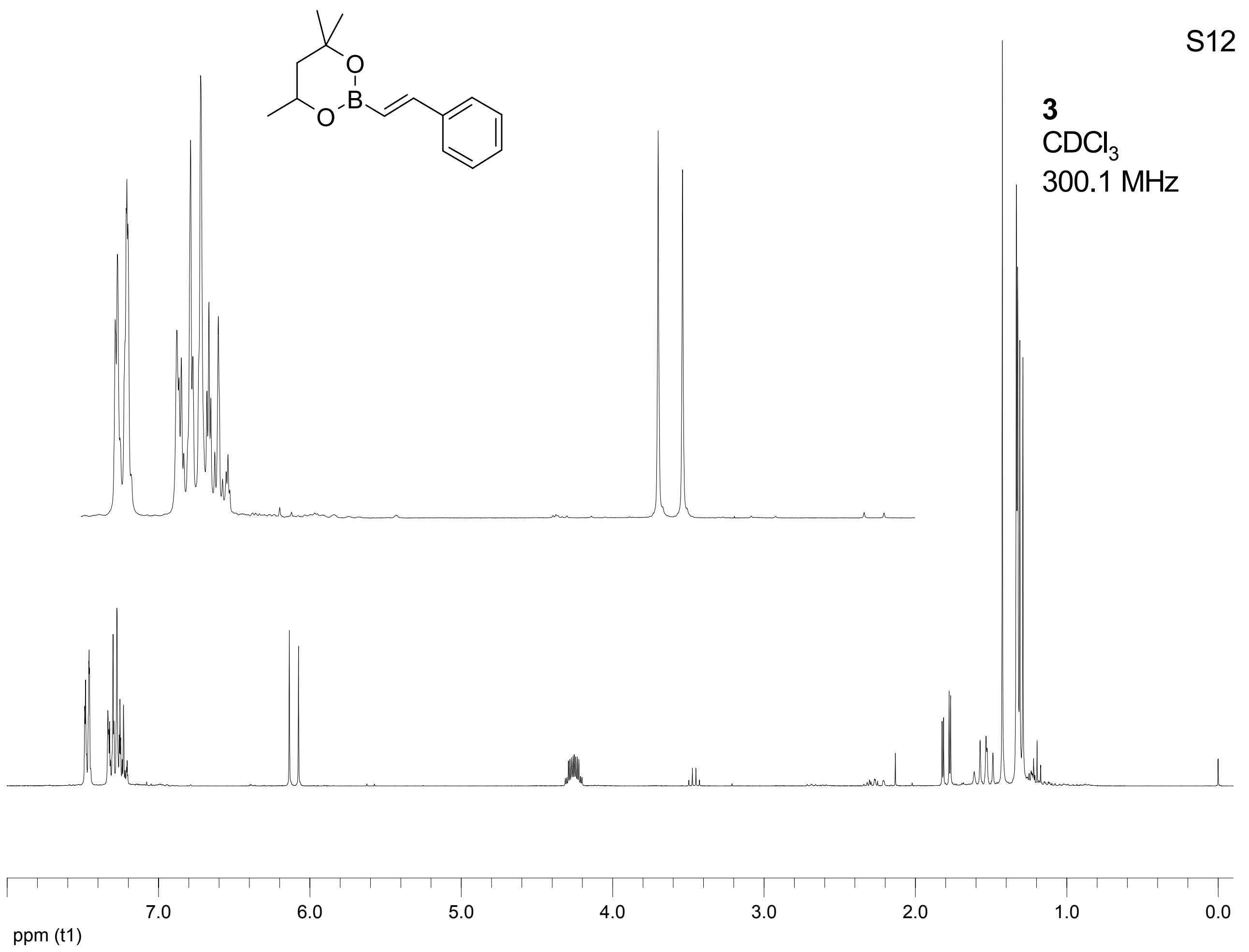


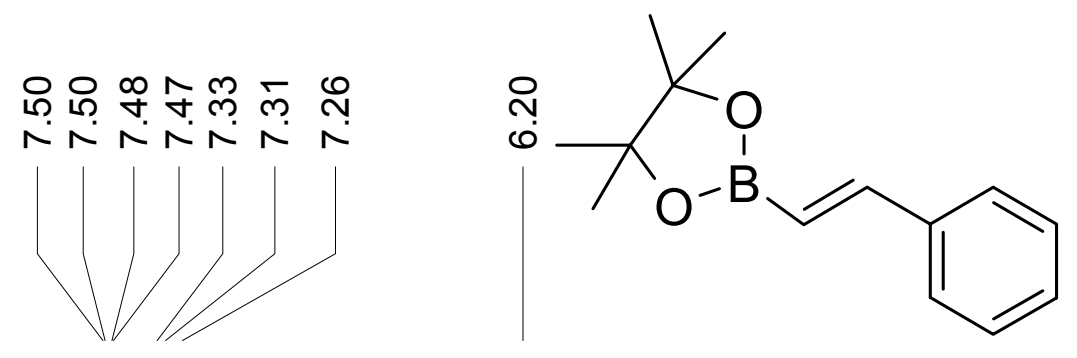

ต़.

$\mathrm{S} 13$

3

$\mathrm{CDCl}_{3}$ $300.1 \mathrm{MHz}$
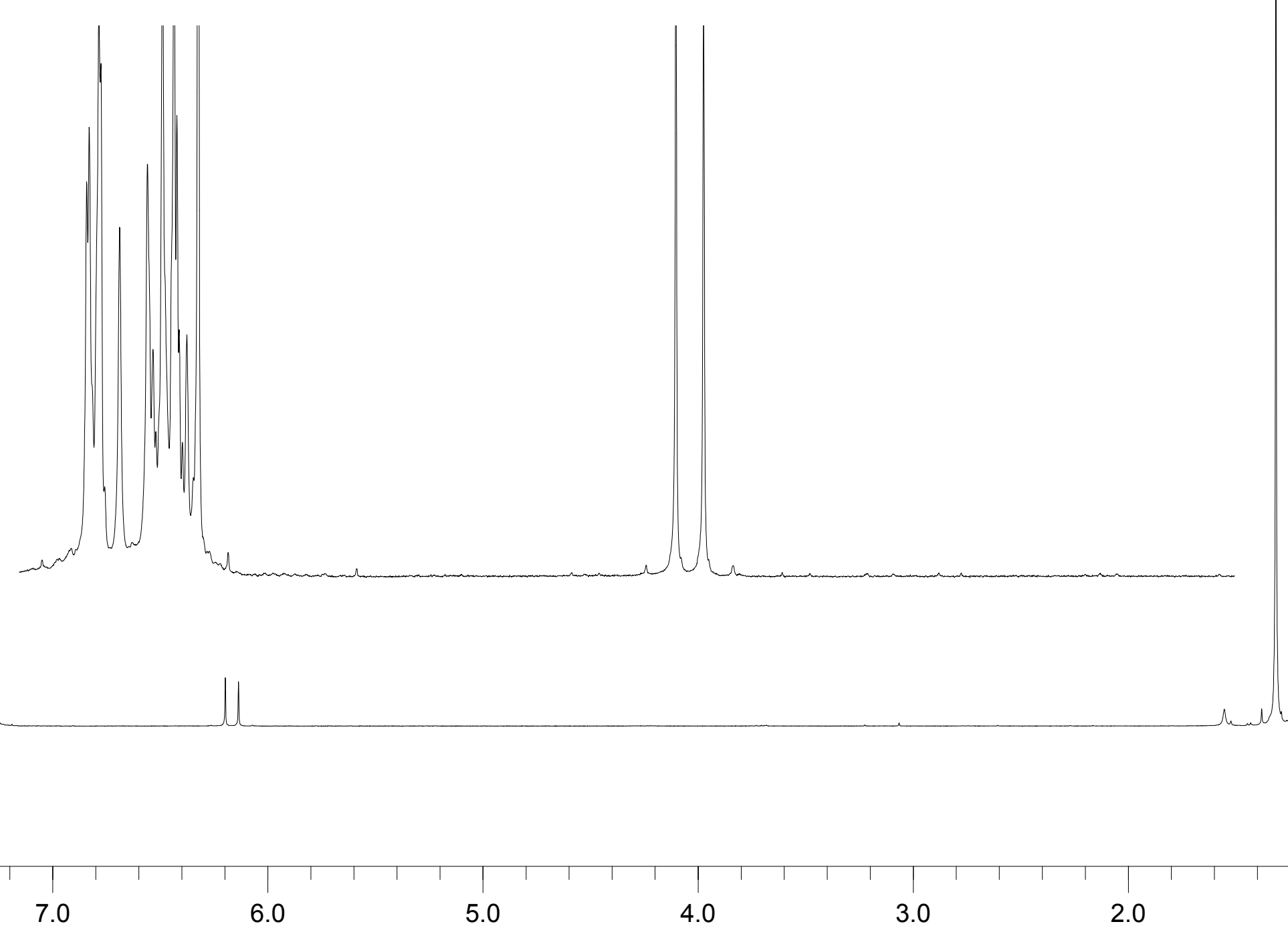

6.0

5.0

4.0

3.0

2.0

1.0

0.0 

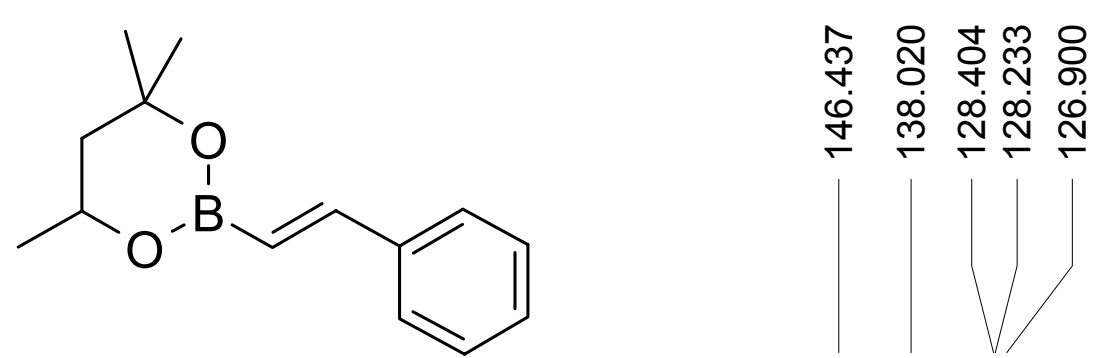

용요

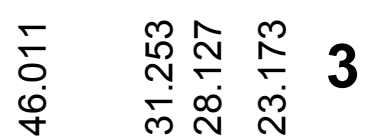

S14

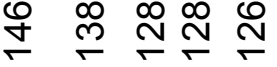

$\mathrm{CDCl}_{3}$

$75.5 \mathrm{MHz}$

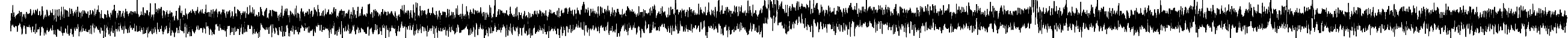




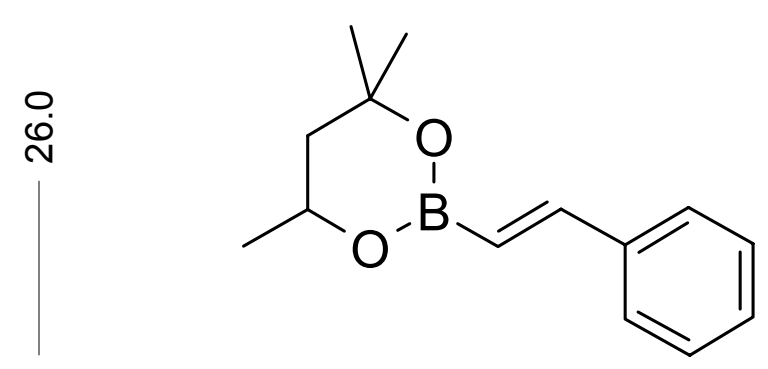

3

$\mathrm{CDCl}_{3}$

$96 \mathrm{MHz}$

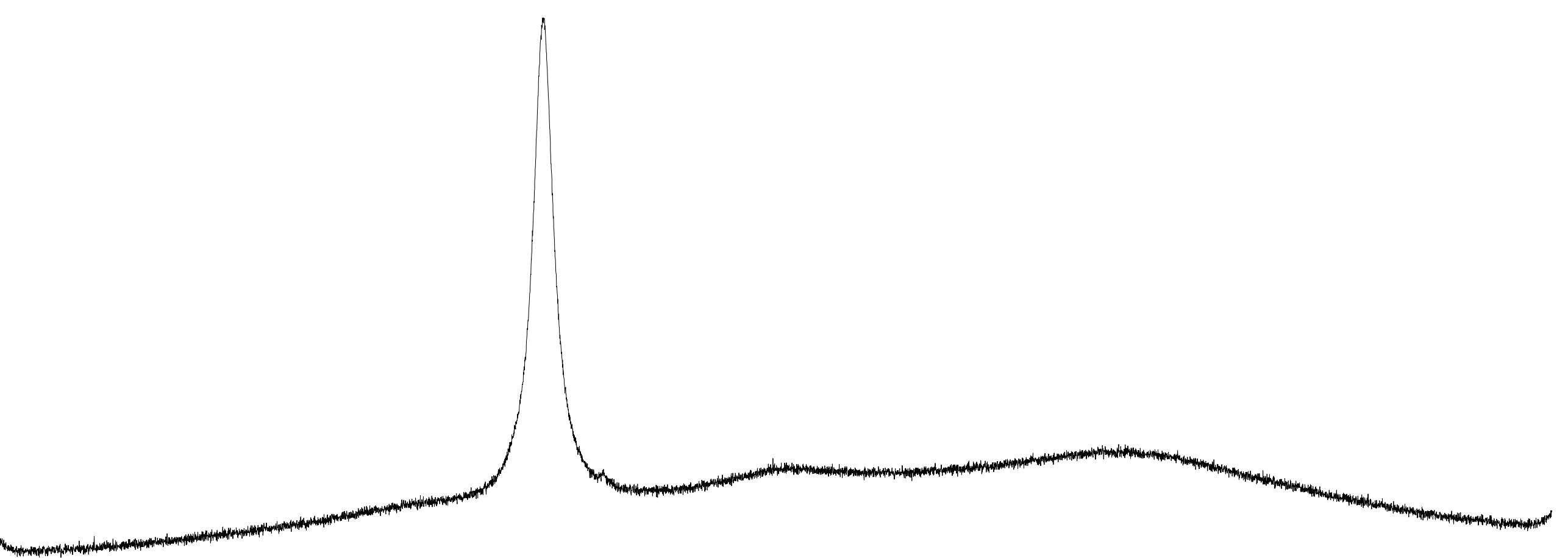




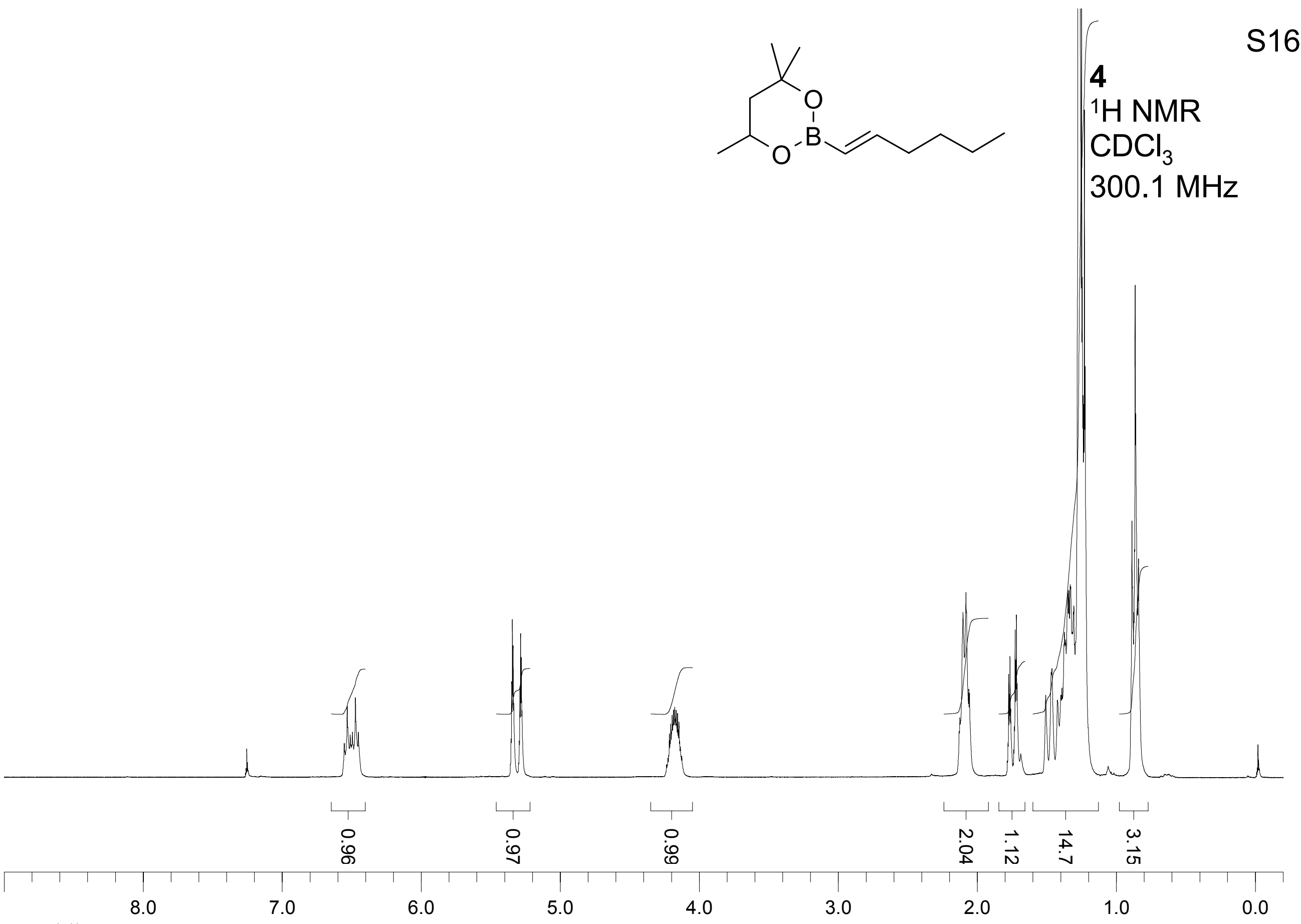


4

${ }^{13} \mathrm{C}$ NMR

$\mathrm{CDCl}_{3}$

$75.5 \mathrm{MHz}$

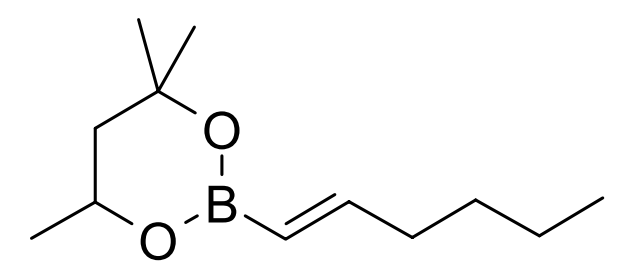

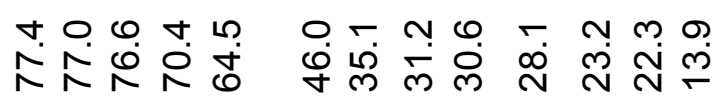

|l 1
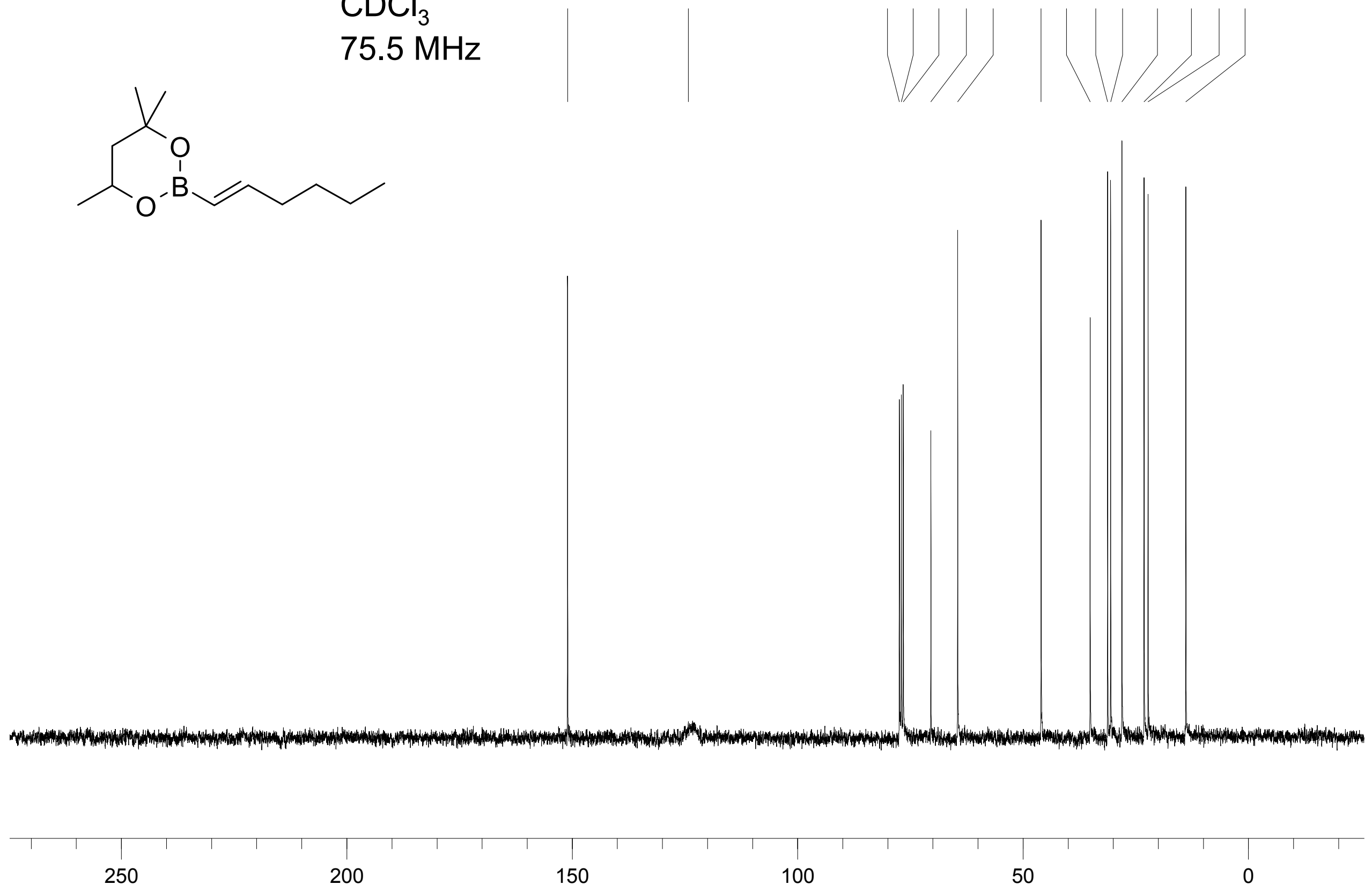

ppm (t1) 


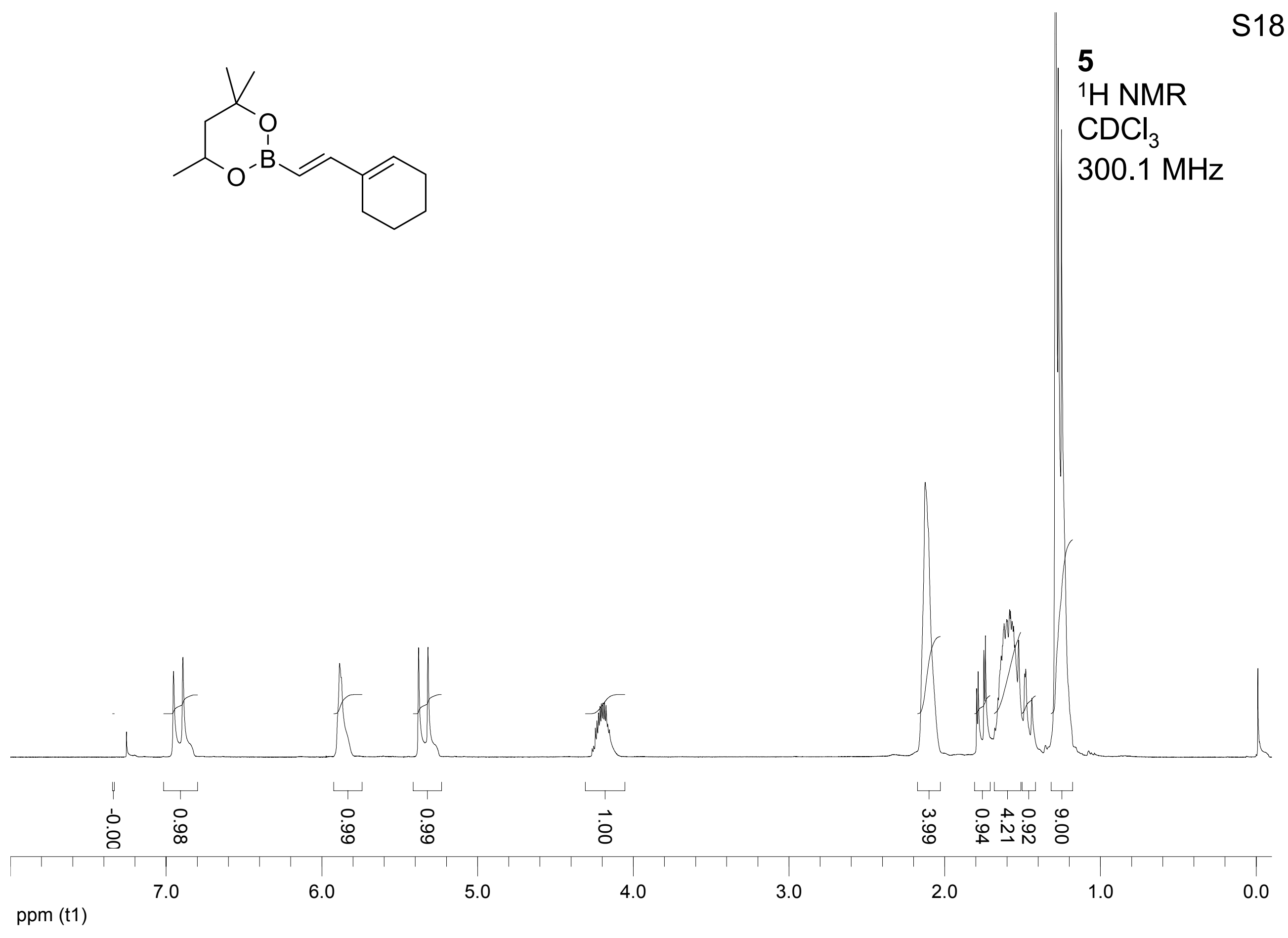




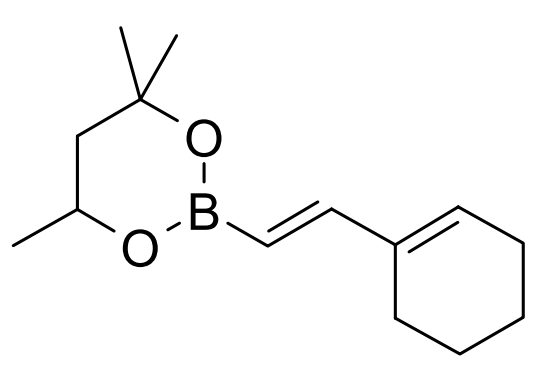

${ }^{13} \mathrm{C}$ NMR

$\mathrm{CDCl}_{3}$

$75.5 \mathrm{MHz}$
일

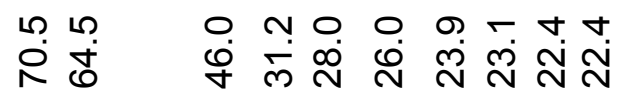

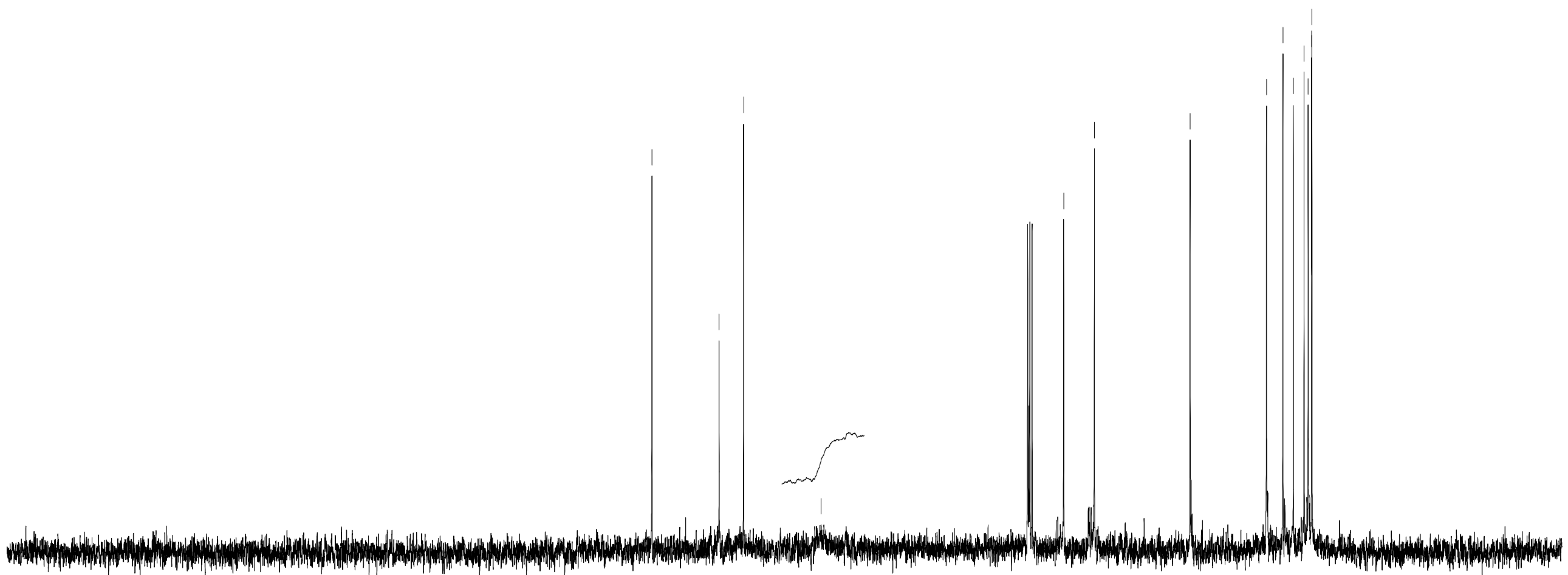




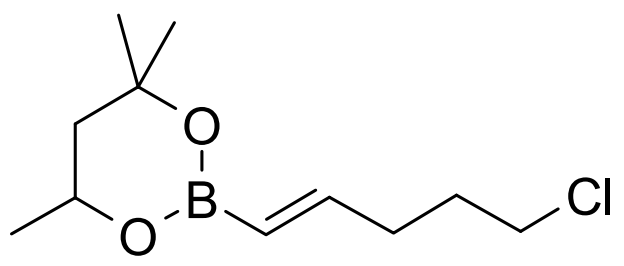

6

${ }^{1} \mathrm{H}$ NMR $\mathrm{CDCl}_{3}$ $300.1 \mathrm{MHz}$

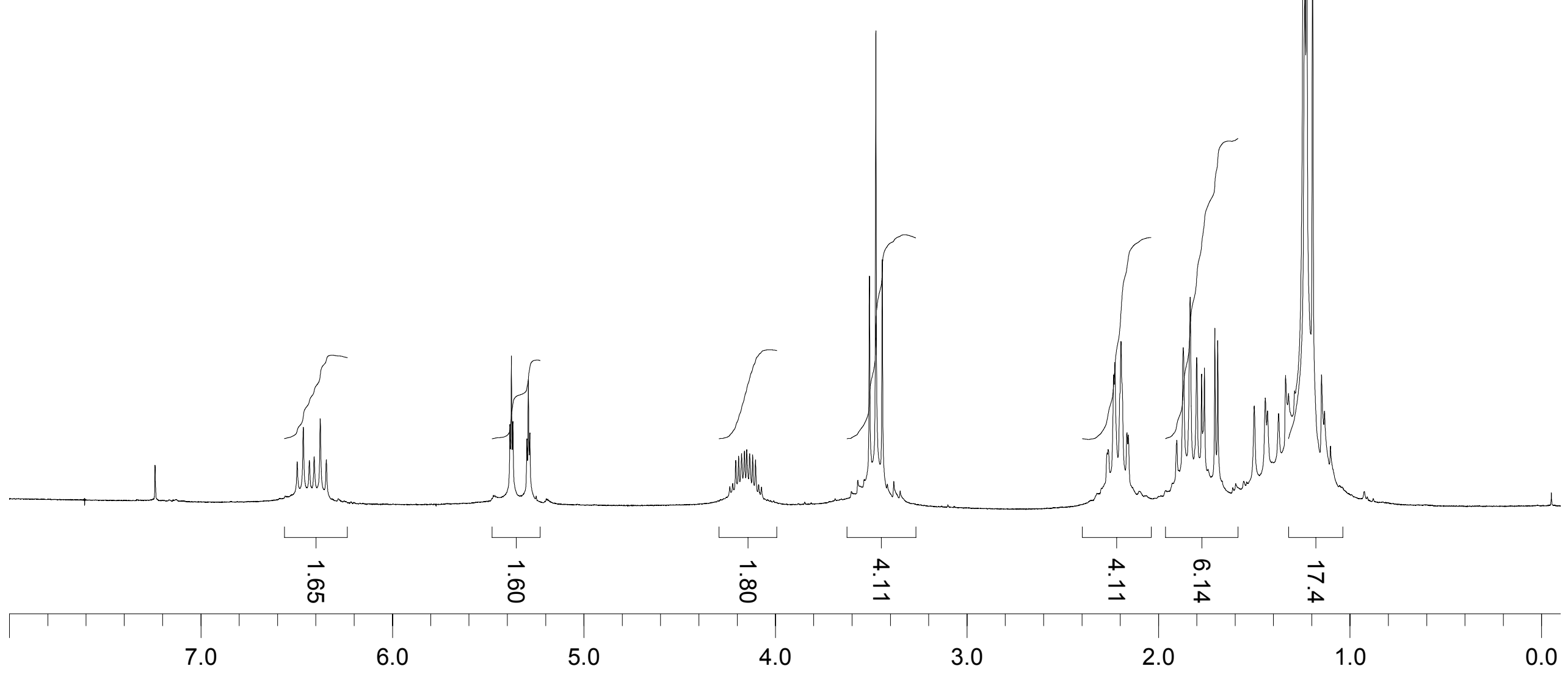

ppm (t1) 


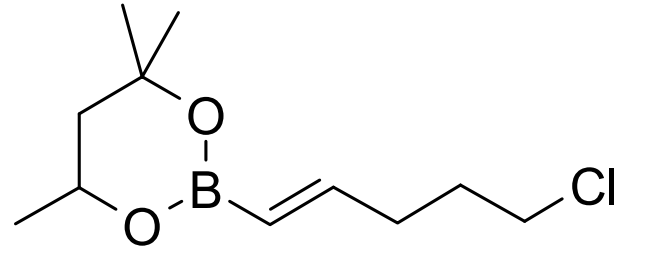

6

${ }^{13} \mathrm{C}$ NMR $\stackrel{\infty}{\mathrm{o}}$

$\mathrm{CDCl}_{3}$

$75.5 \mathrm{MHz}$

${ }^{13} \mathrm{C}, 75.5 \mathrm{MHz}, \mathrm{CDCl}_{3}$

$\stackrel{\sim}{\stackrel{N}{\sim}}$

in

ब. m m ก -

守 寸

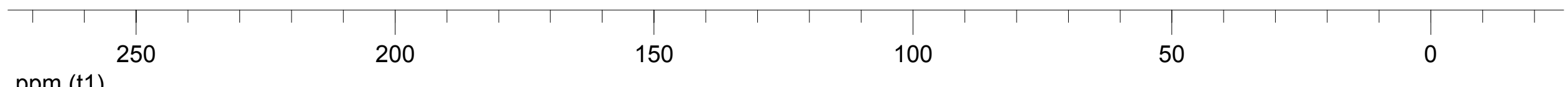

ppm (t1) 

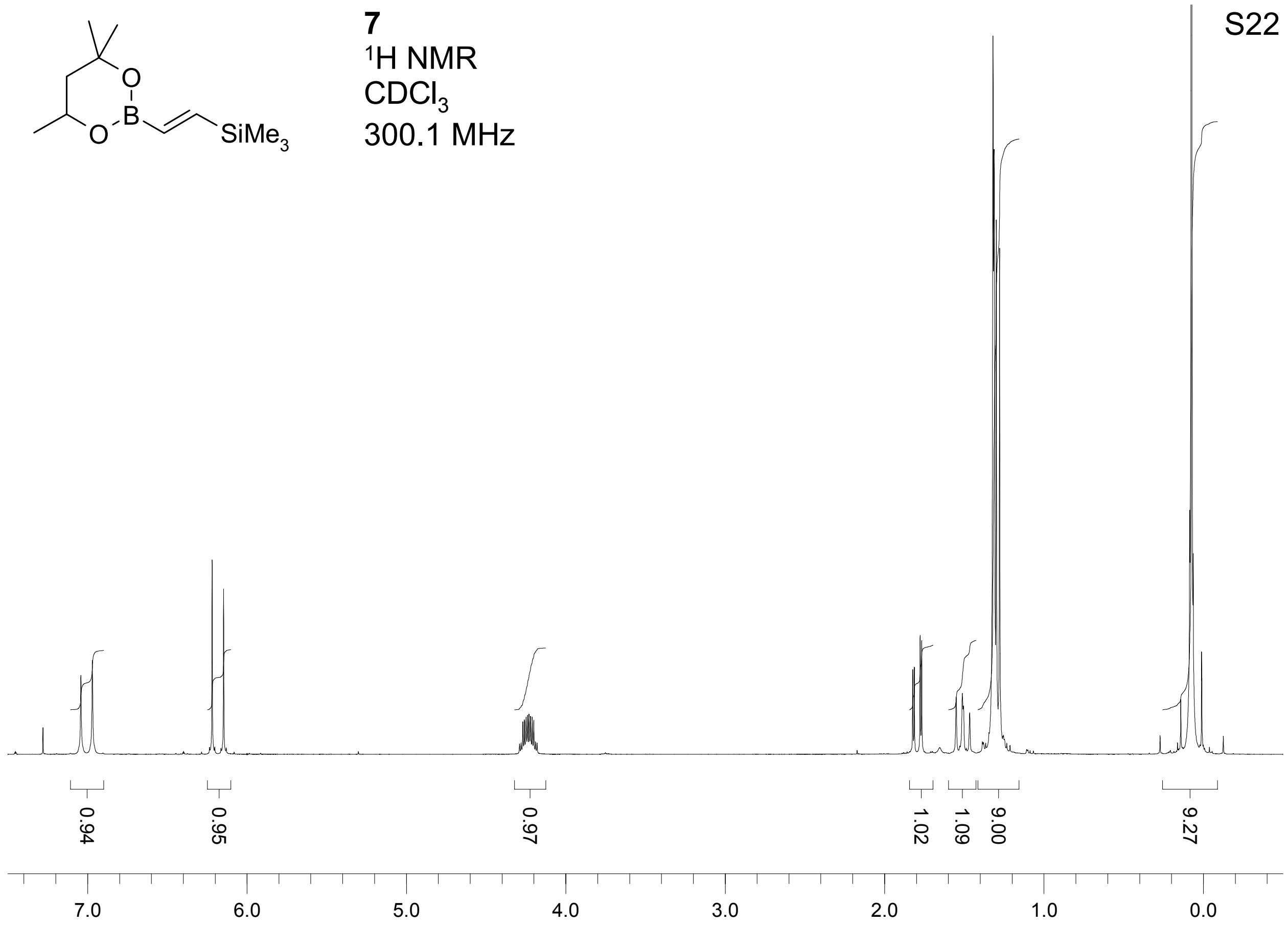


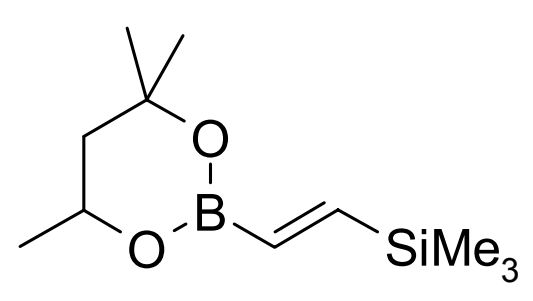

${ }^{13} \mathrm{C} \mathrm{NMR}^{2}$
$\mathrm{CDCl}_{3}$

实守

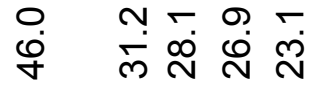

$75.5 \mathrm{MHz}$
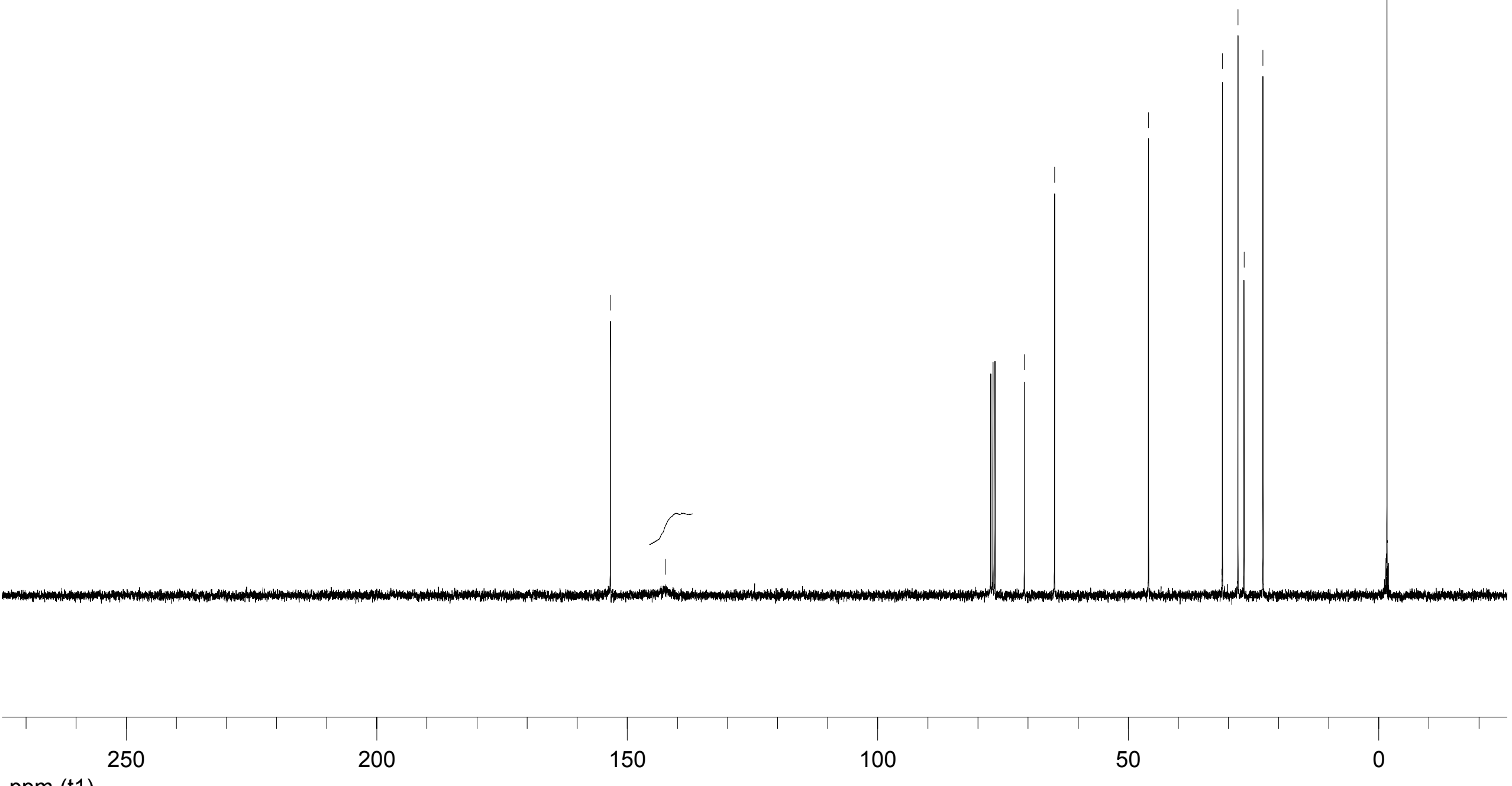

ppm (t1) 


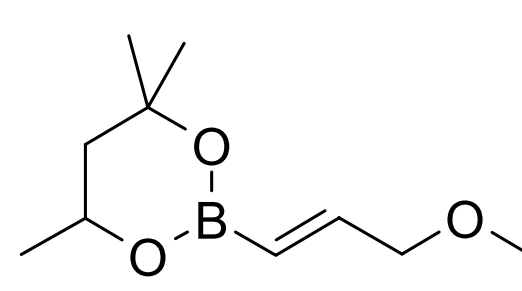

8

${ }^{1} \mathrm{H}$ NMR

$\mathrm{CDCl}_{3}$

$300.1 \mathrm{MHz}$

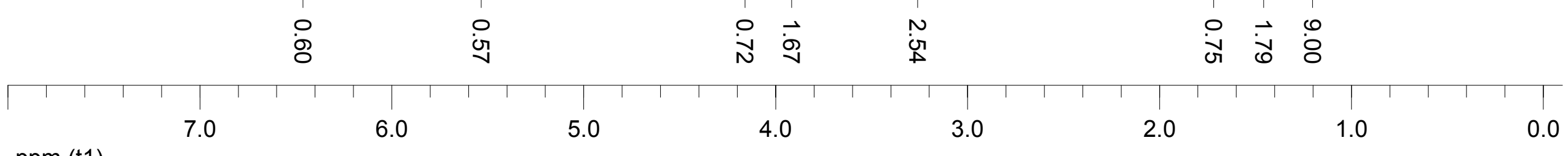

ppm (t1) 


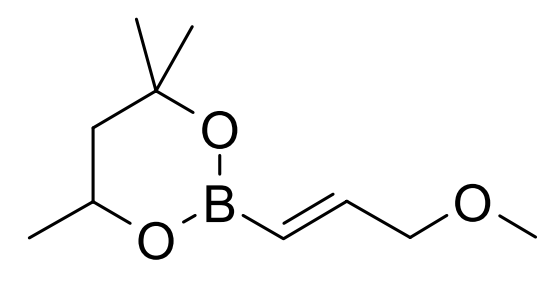

8

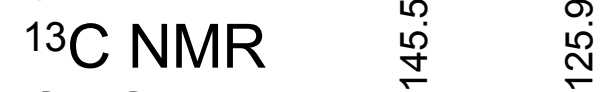

$\mathrm{CDCl}_{3}$

$75.5 \mathrm{MHz}$

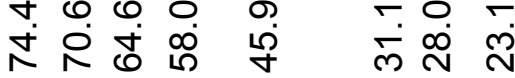

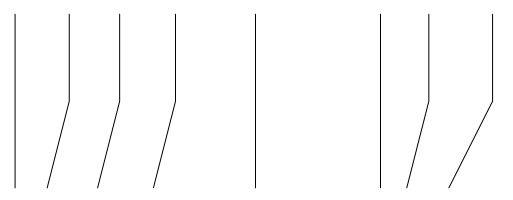

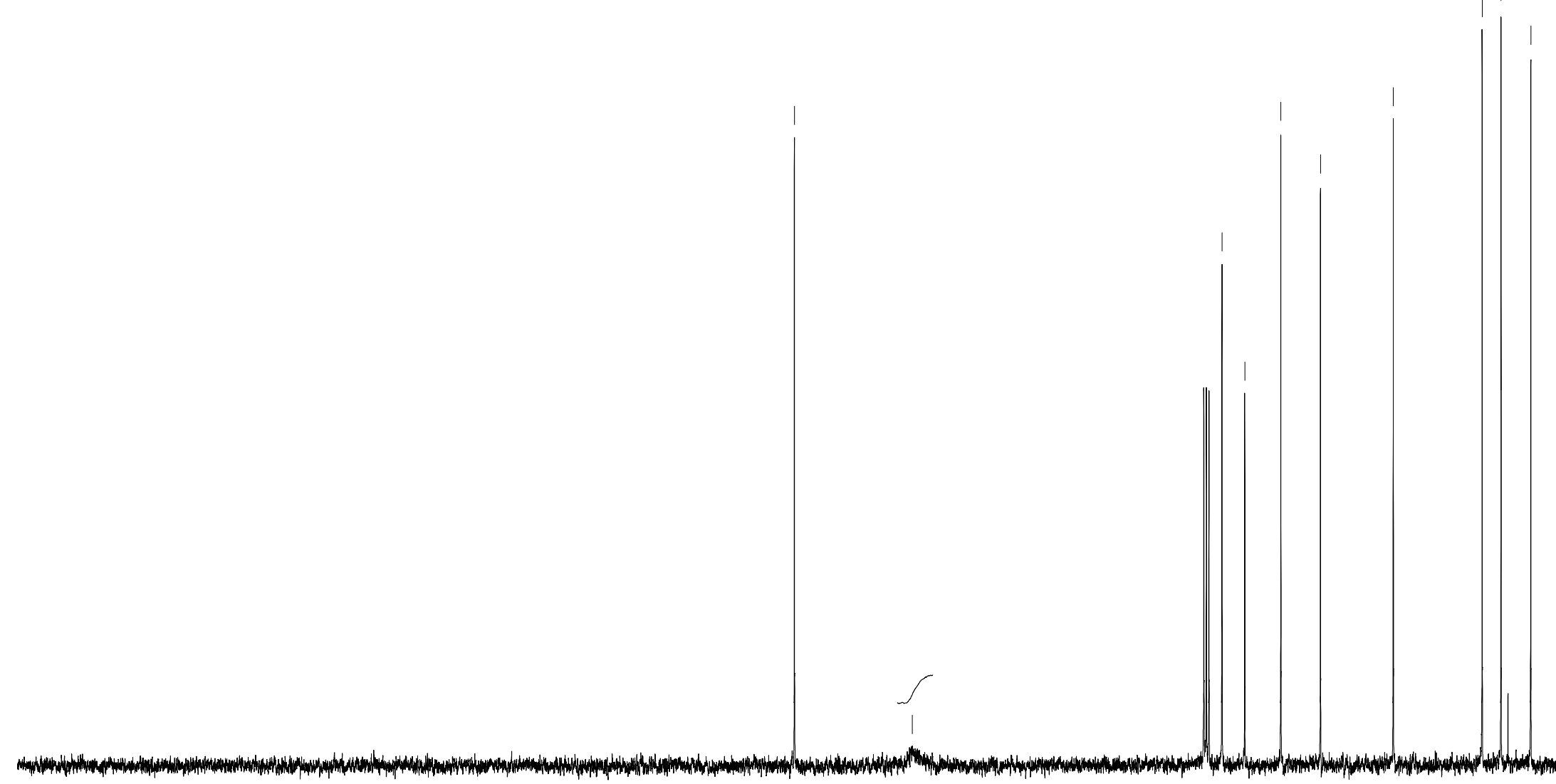

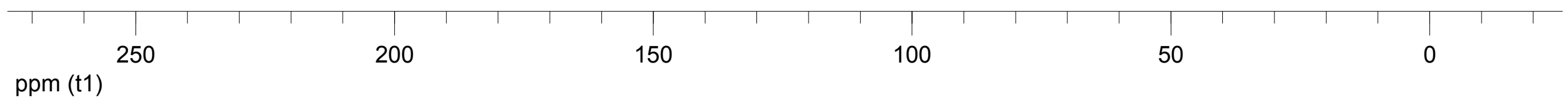



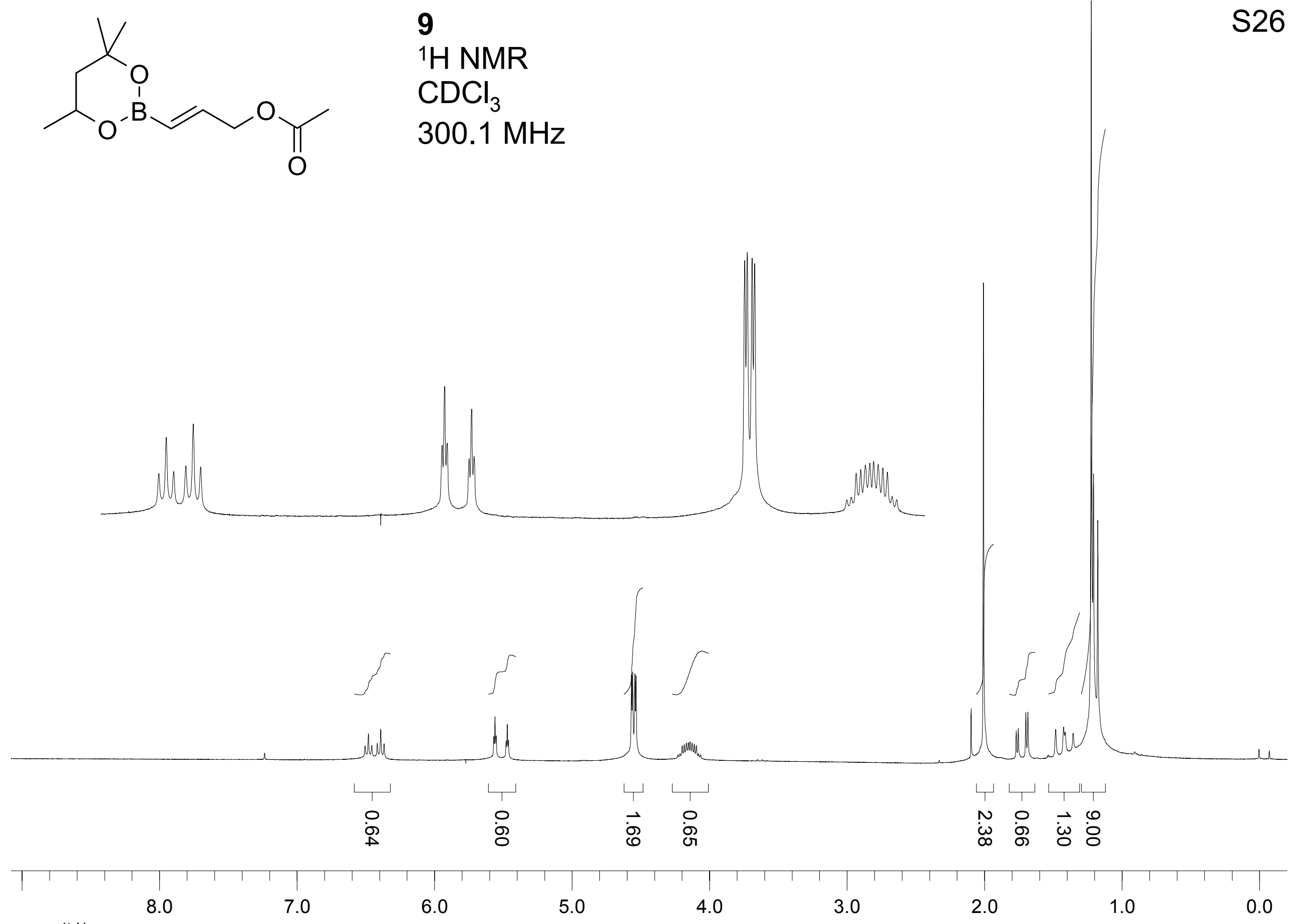

ppm (t1) 
<smiles>CC(=O)OC/C=C/B1OC(C)CC(C)(C)O1</smiles>

9

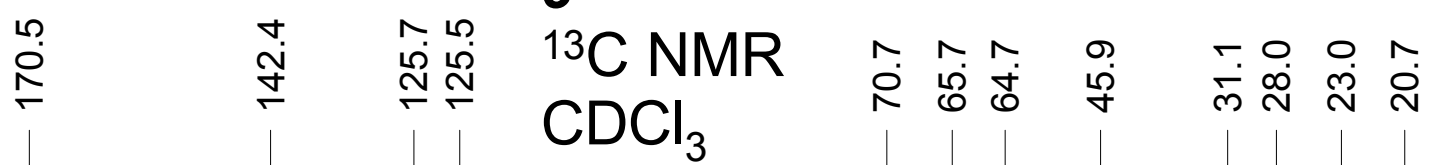
$75.5 \mathrm{MHz}$

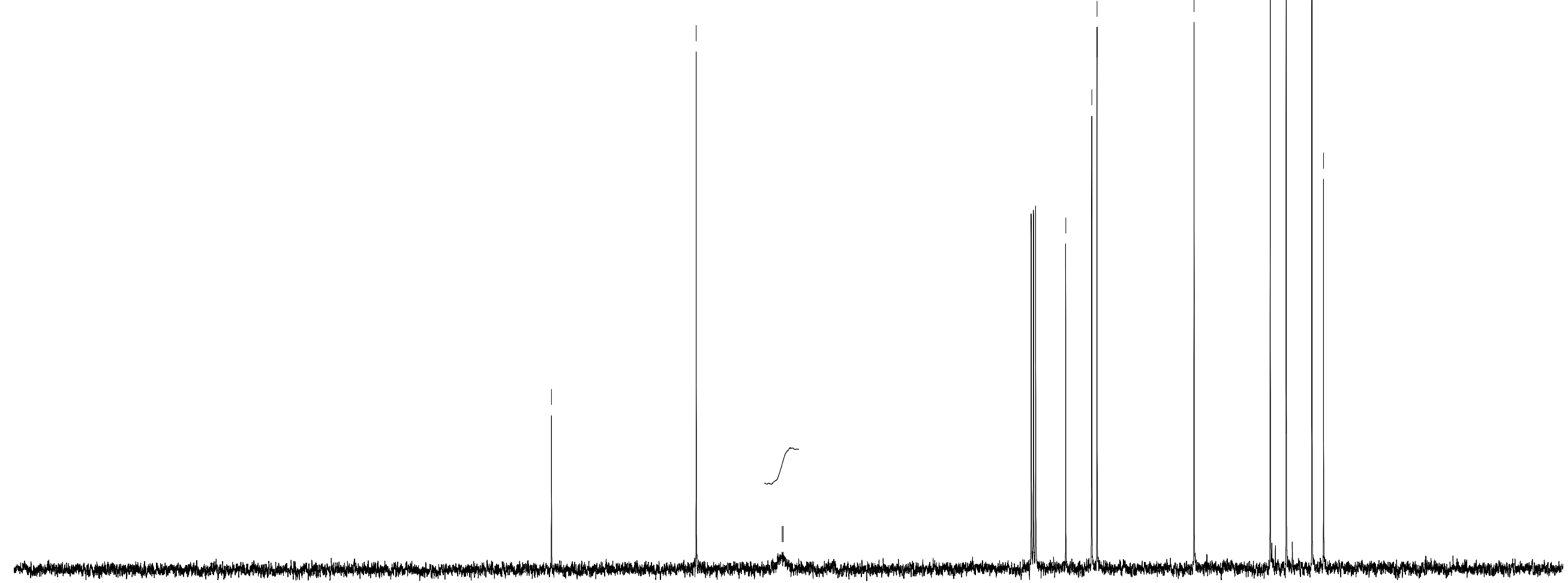

ppm (t1) 


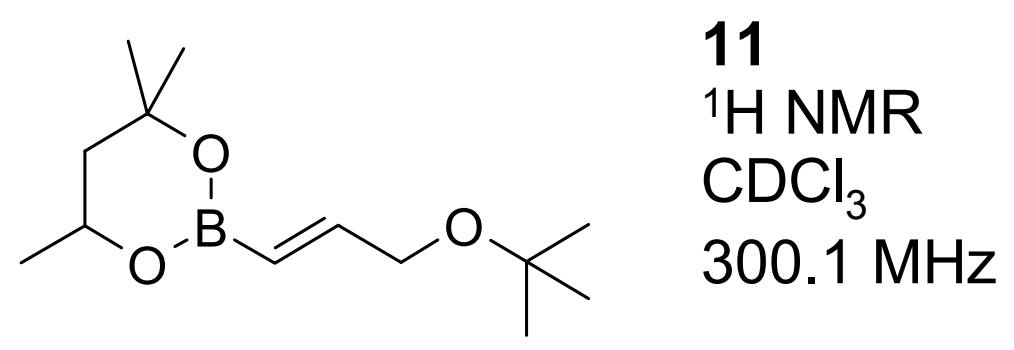

S28

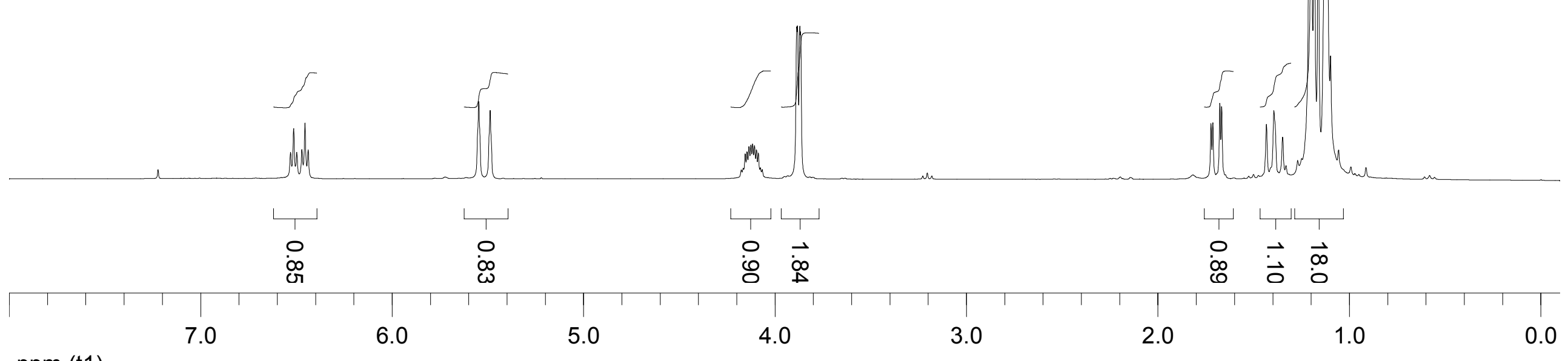




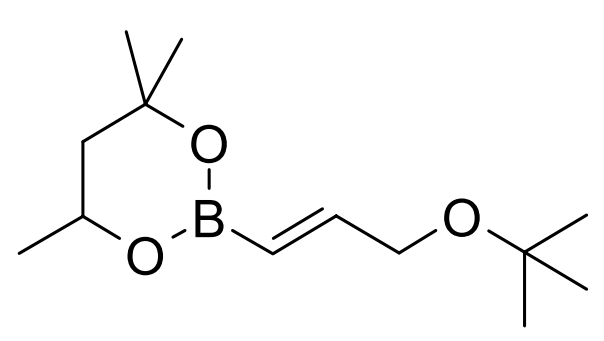

${ }^{13} \mathrm{C}$ NMR

$\stackrel{m}{\stackrel{9}{+}}$

S29 $\mathrm{CDCl}_{3}$

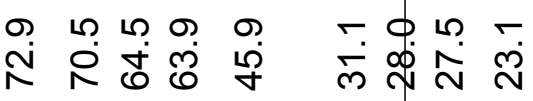
$75.5 \mathrm{MHz}$ 

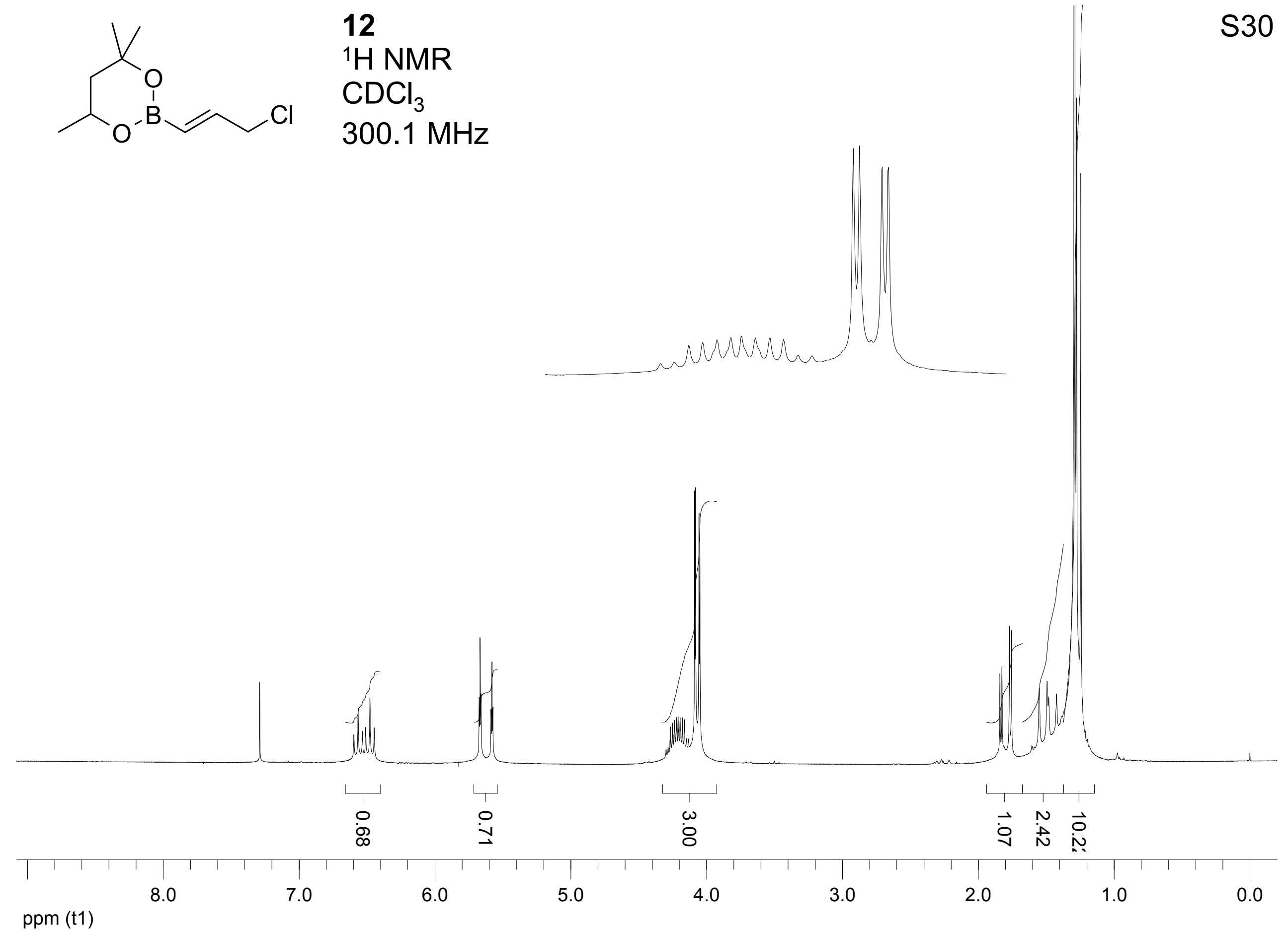


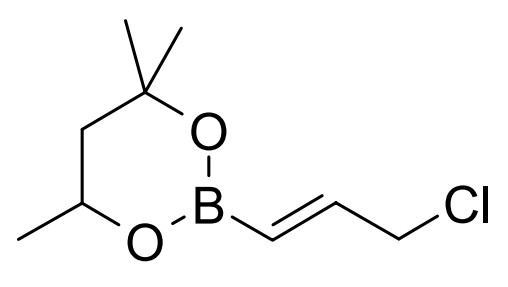
$75.5 \mathrm{MHz}$

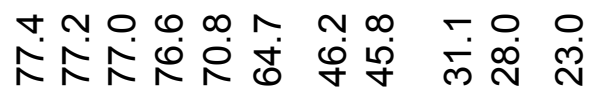
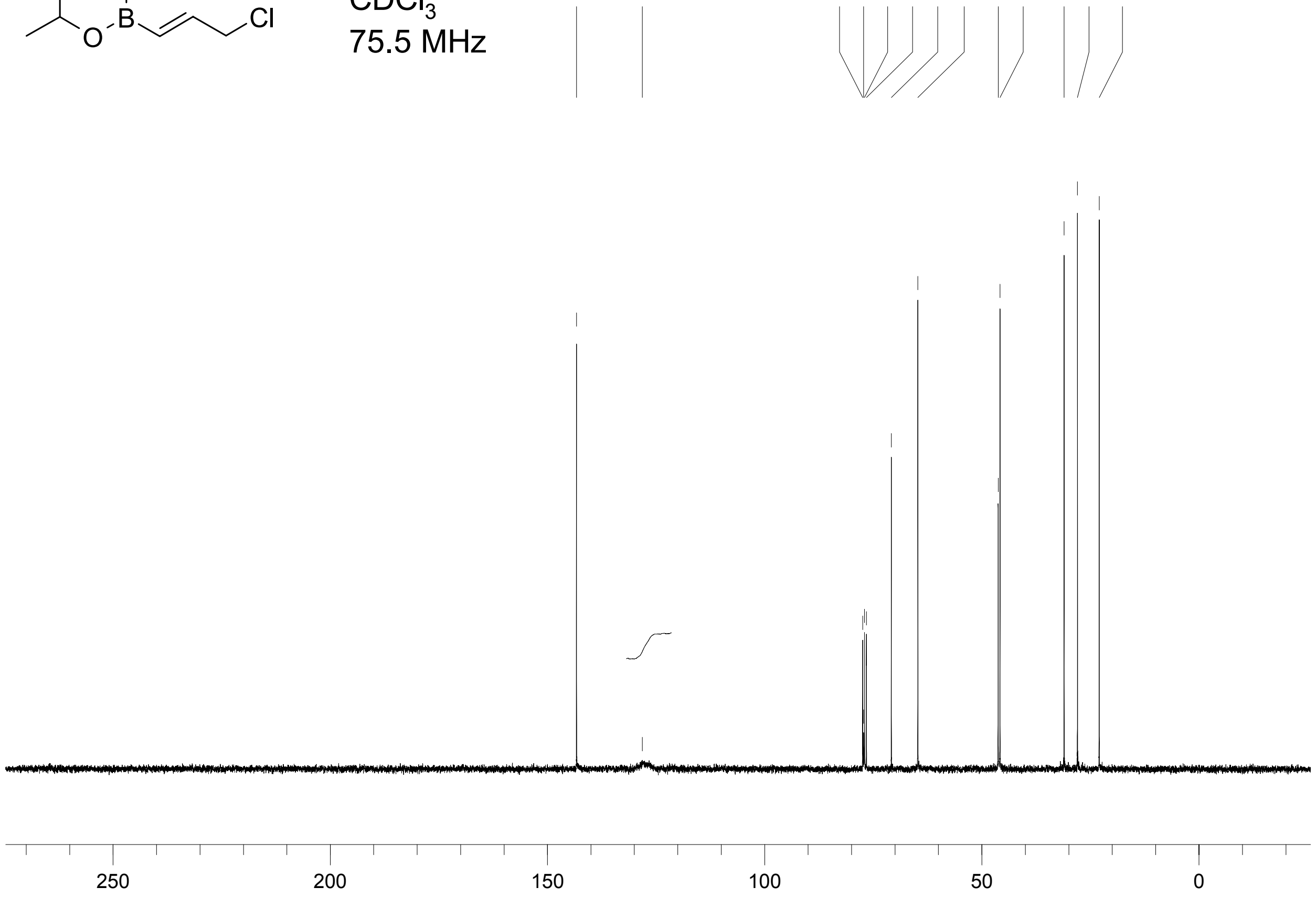

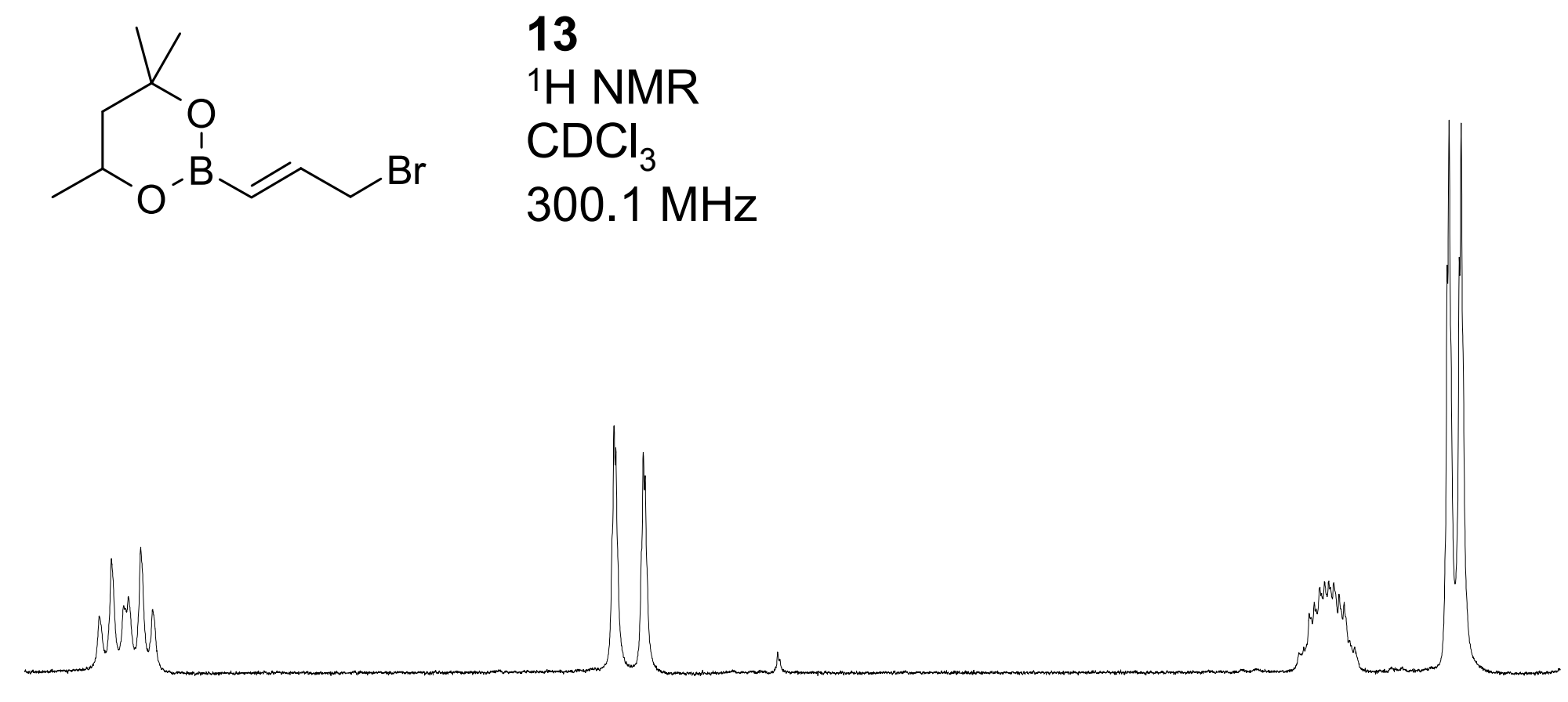

S32

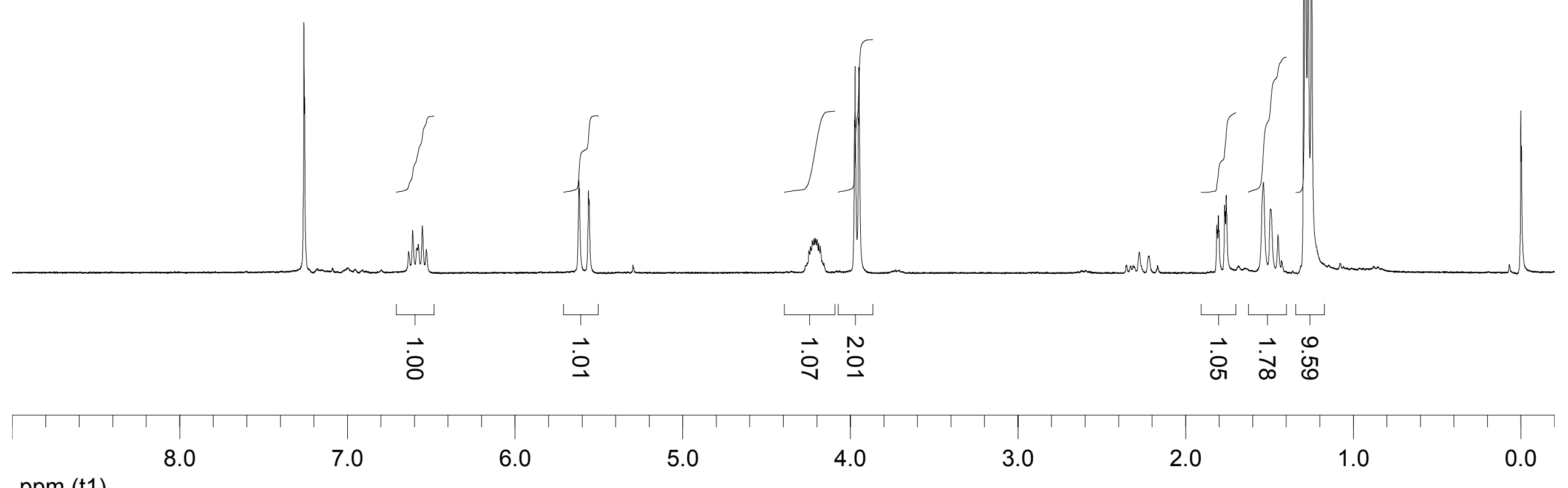




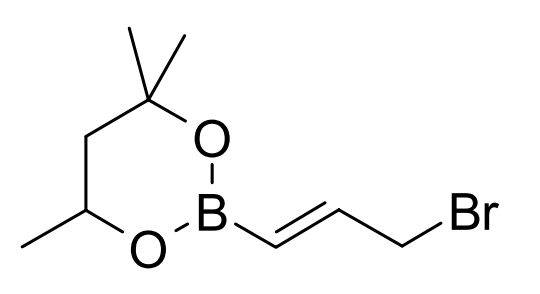

${ }^{13} \mathrm{C}$ NMR

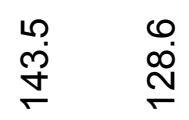

롱요

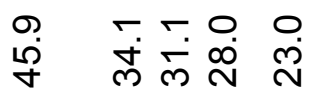

$\mathrm{CDCl}_{3}$

$75.5 \mathrm{MHz}$

S33
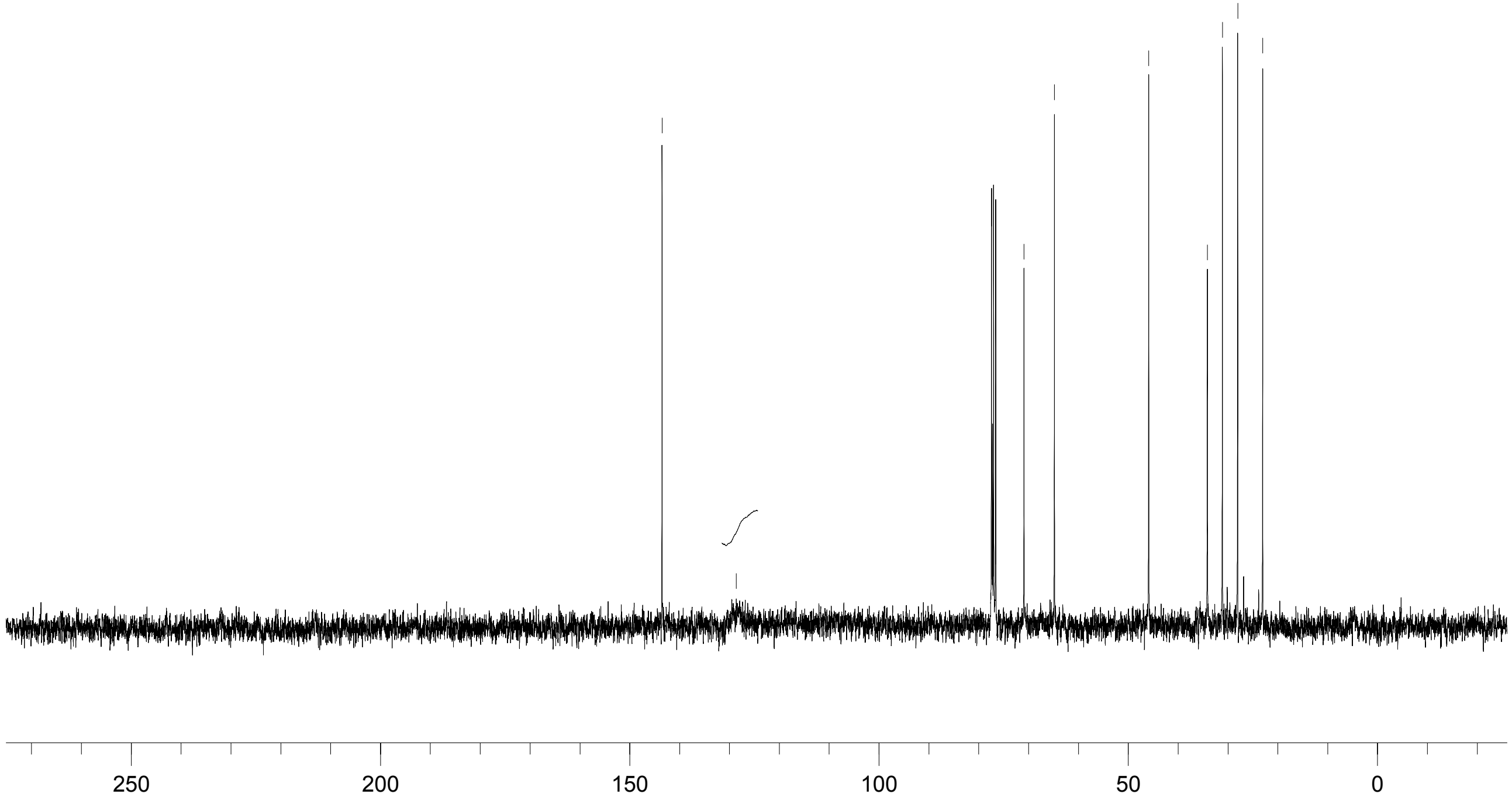

ppm (t1) 


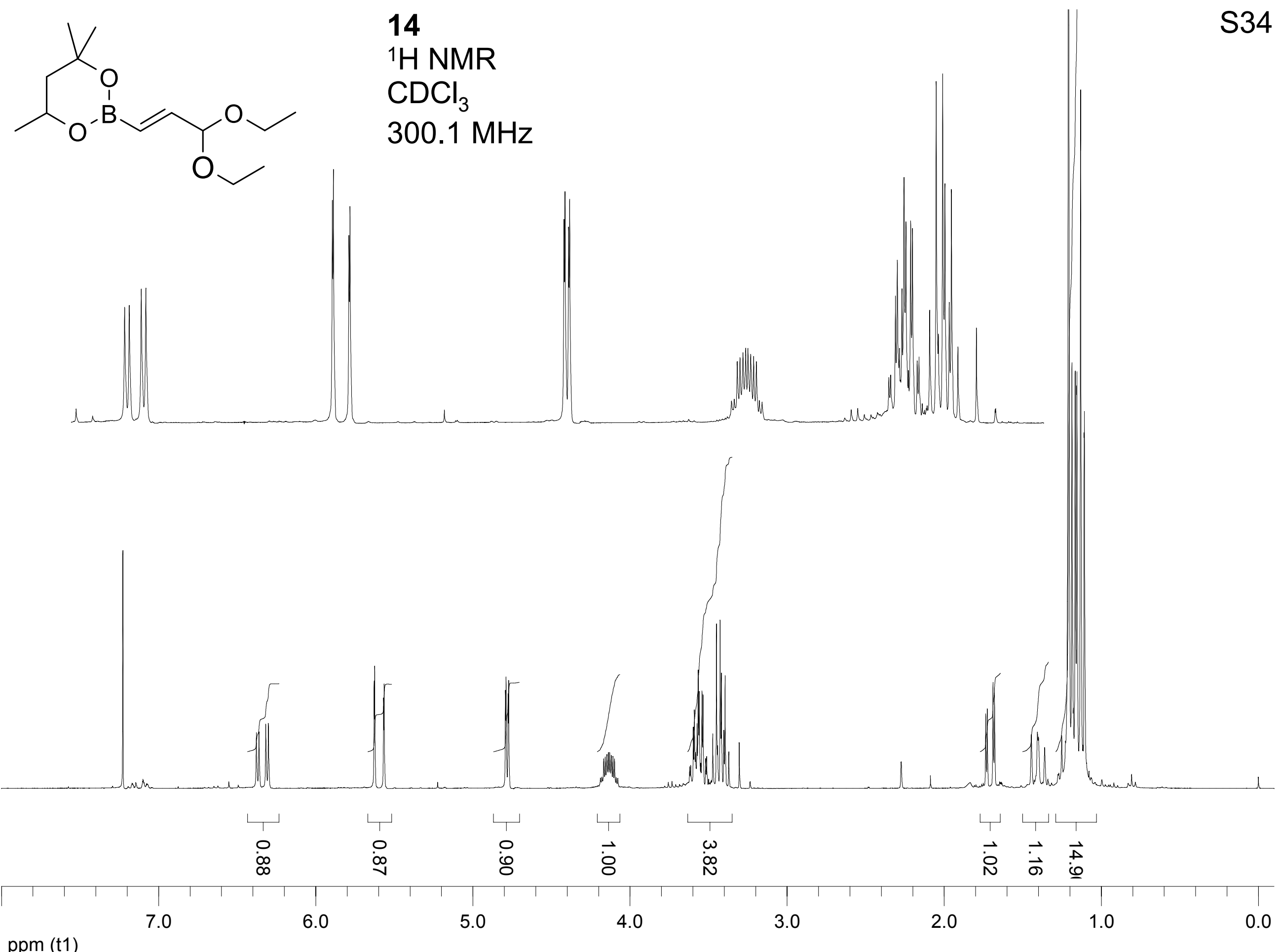




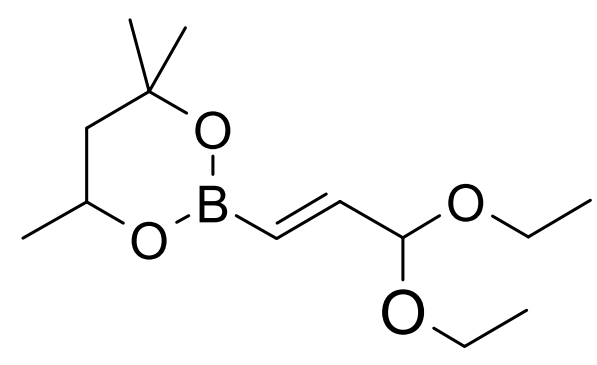
$\mathrm{CDCl}_{3}$ $75.5 \mathrm{MHz}$

75.5

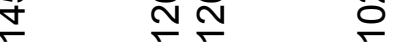

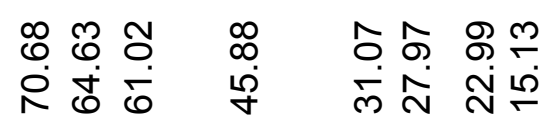

S35
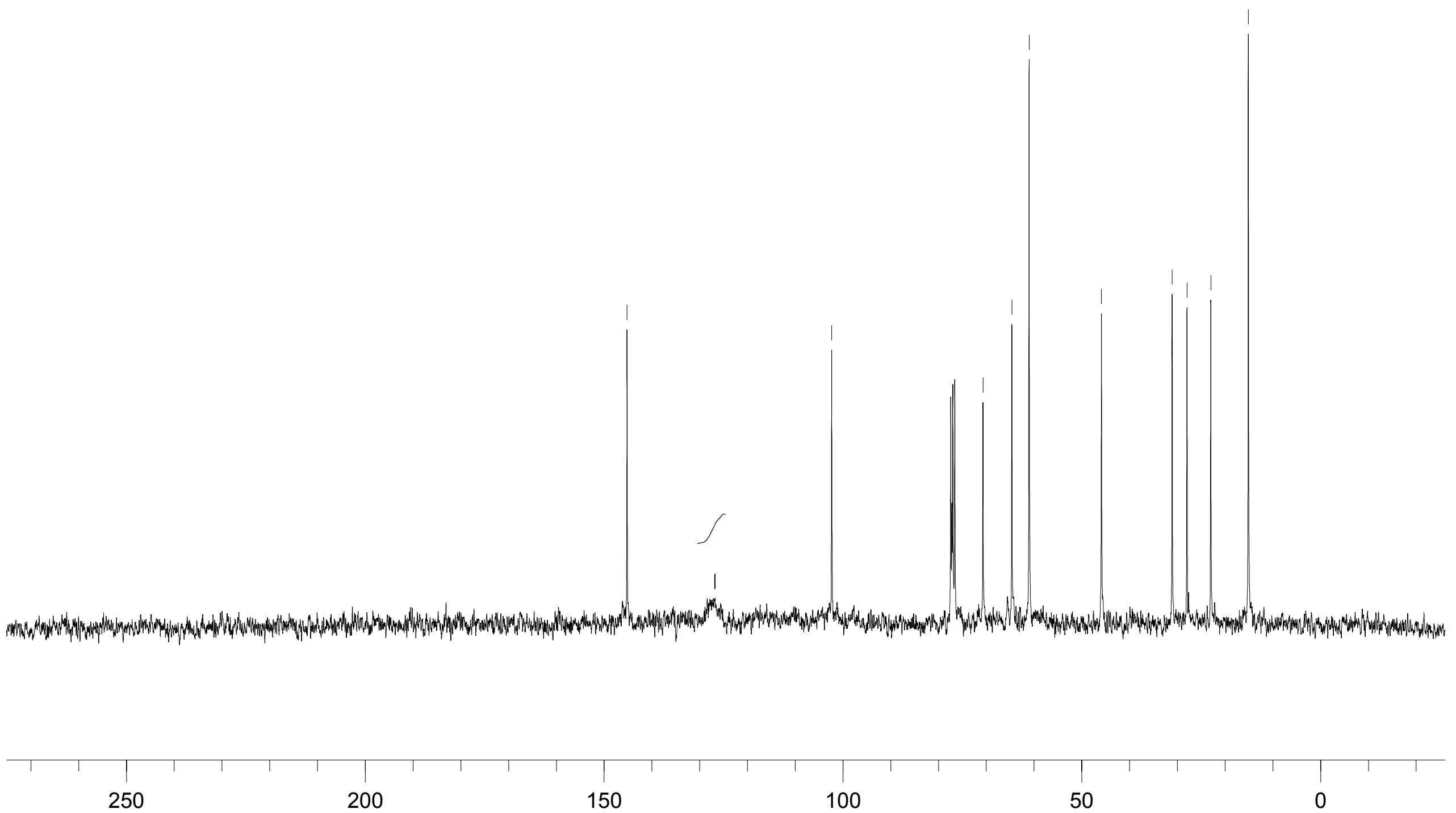

250

200

150

100

50

0

ppm (t1) 


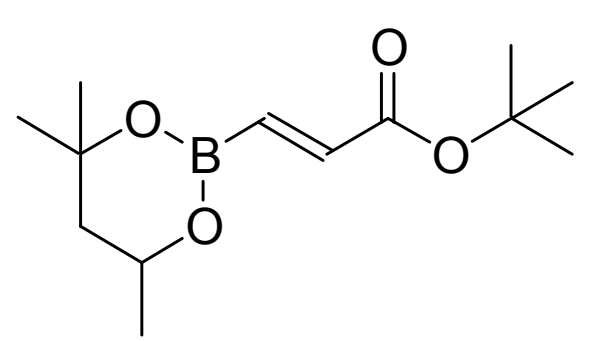

15

${ }^{1} \mathrm{H}$ NMR

$\mathrm{CDCl}_{3}$ 300.1 MHz

${ }^{1} \mathrm{H}, 300.1 \mathrm{MHz}, \mathrm{CDCl}_{3}$

S36

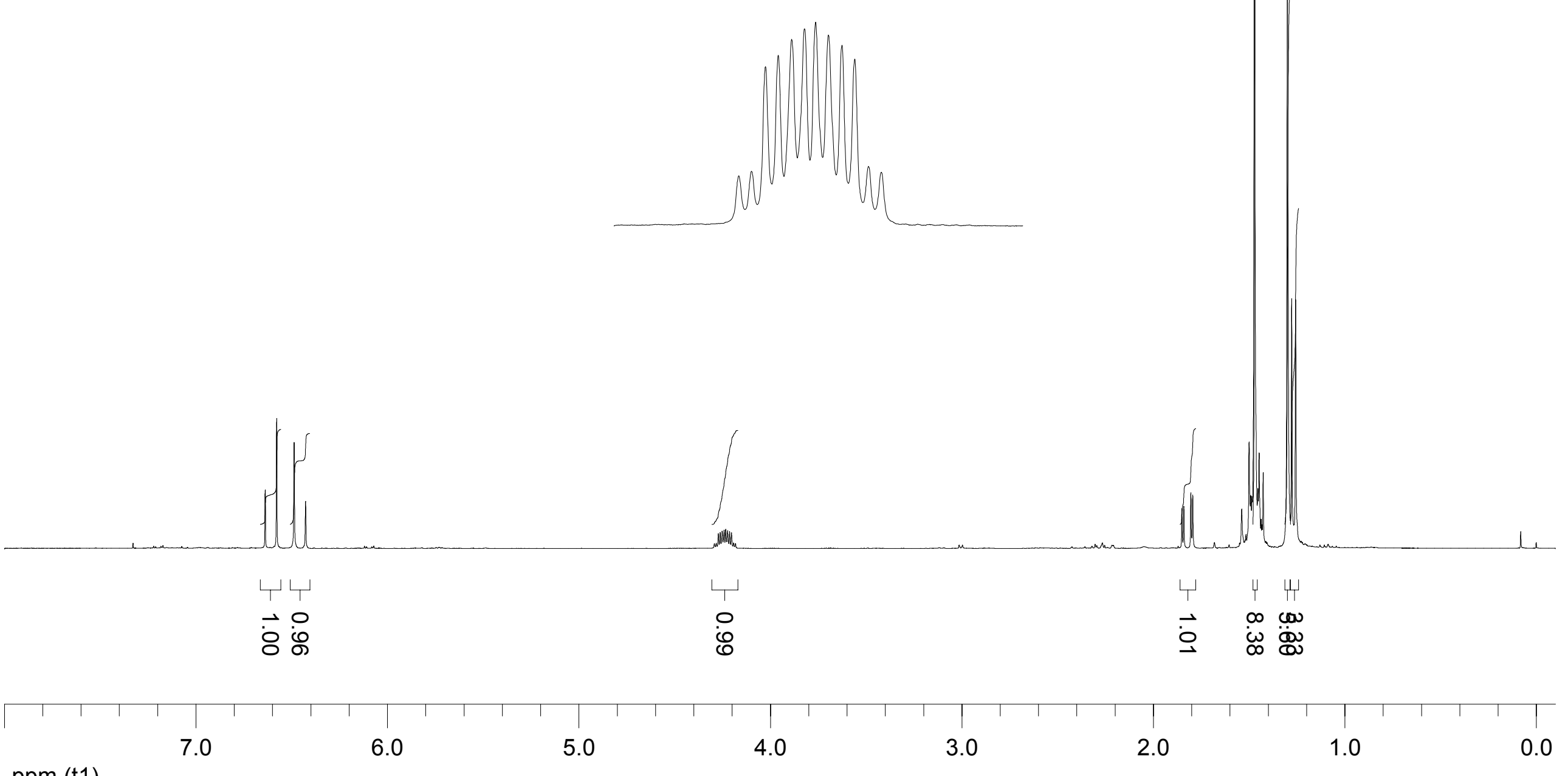

ppm (t1) 

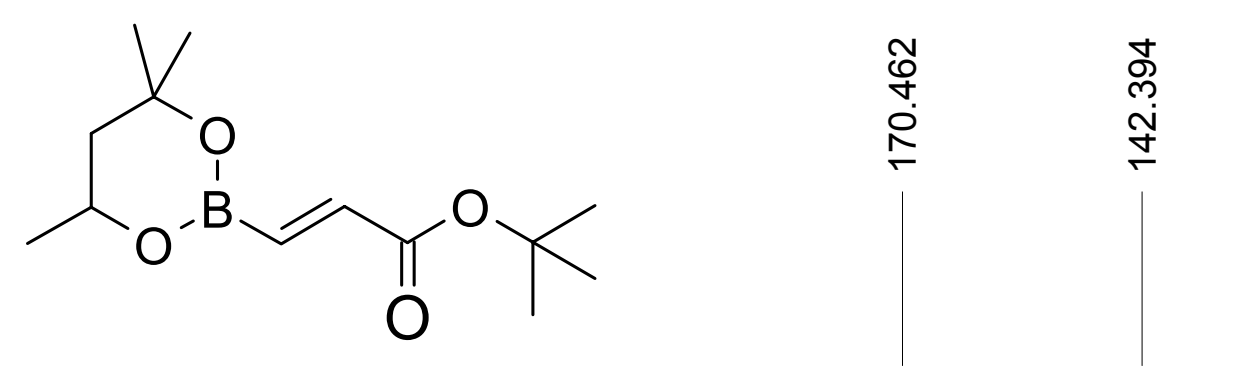

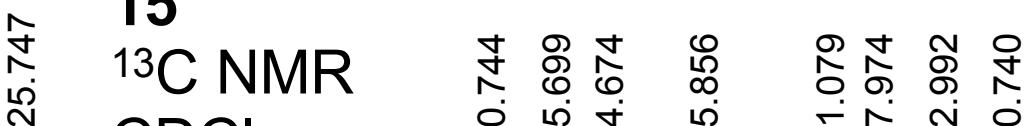
$\mathrm{CDCl}_{3}$

एँ $75.5 \mathrm{MHz}$
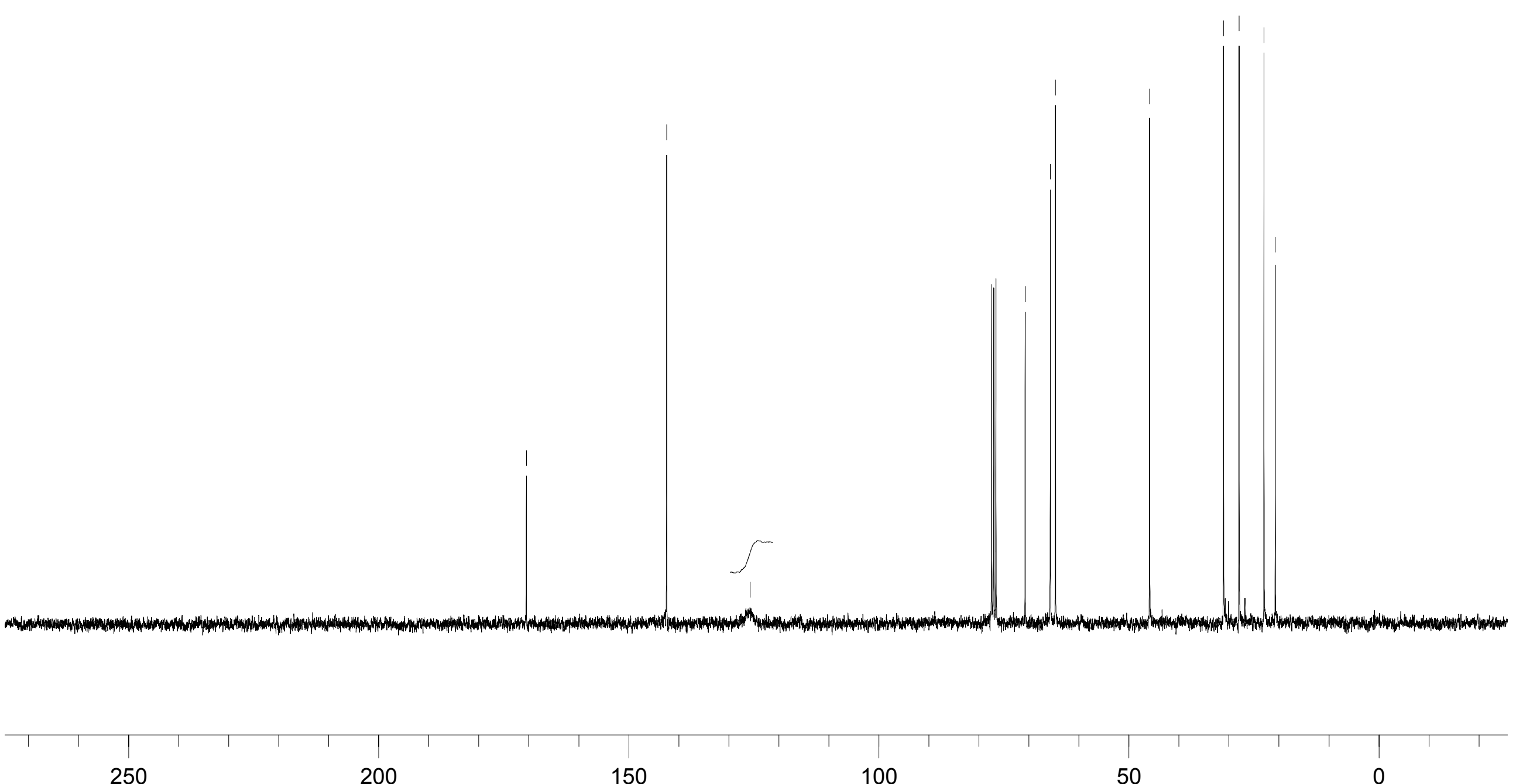

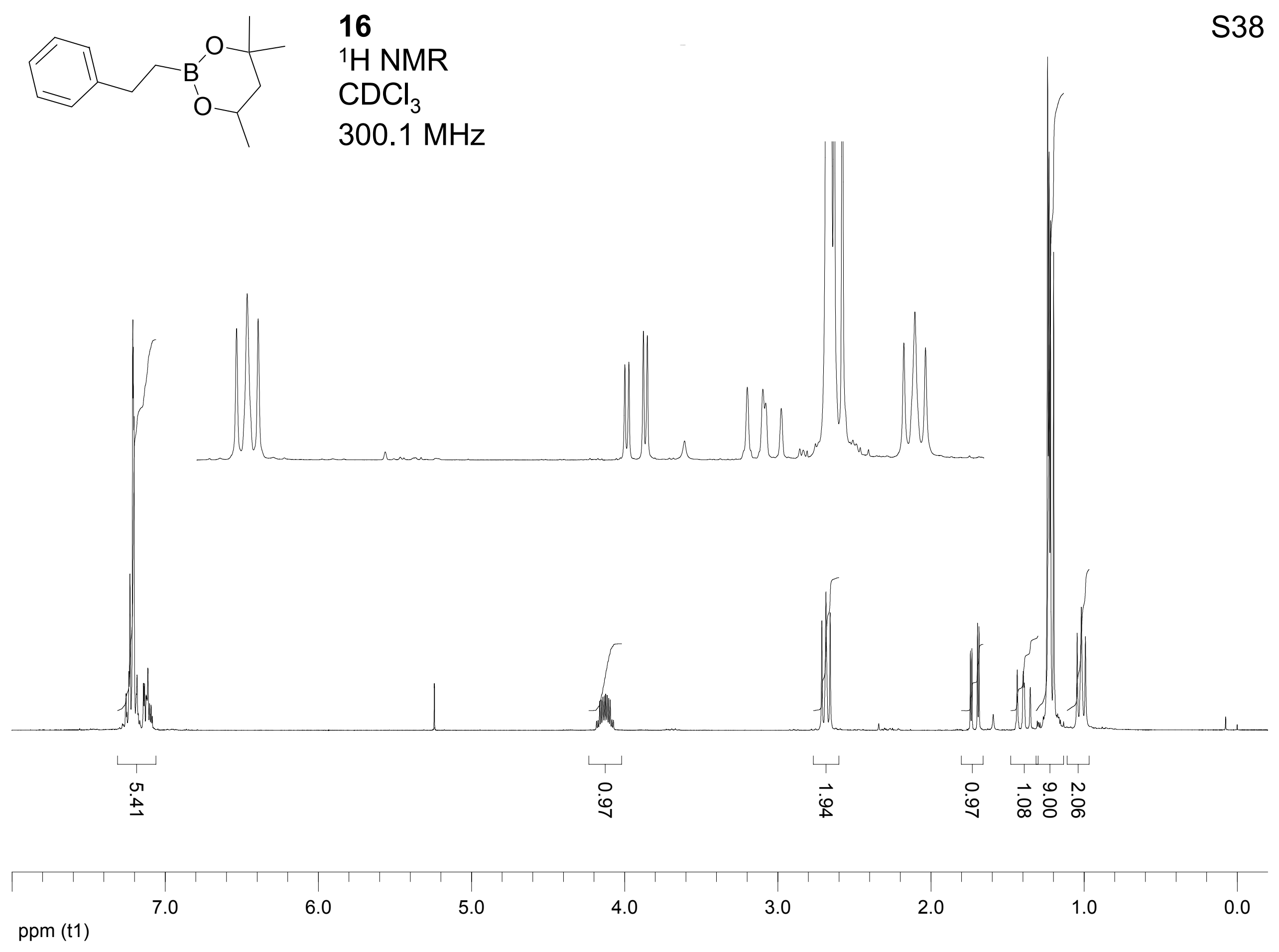


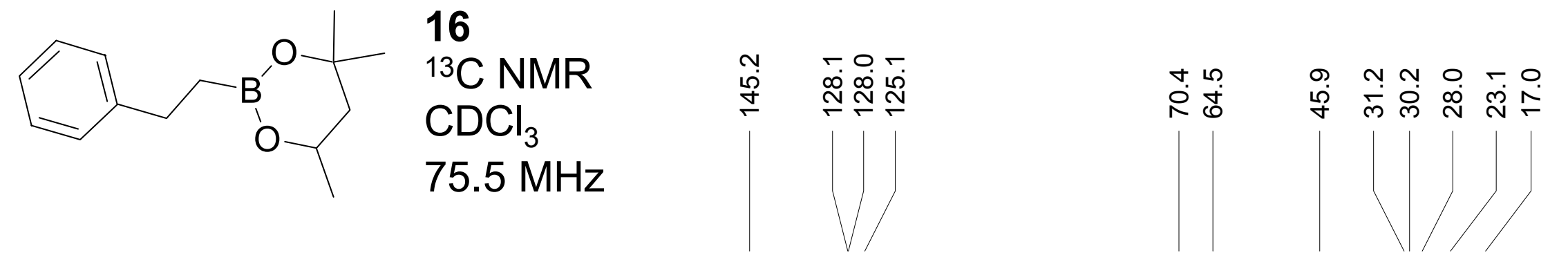

S39
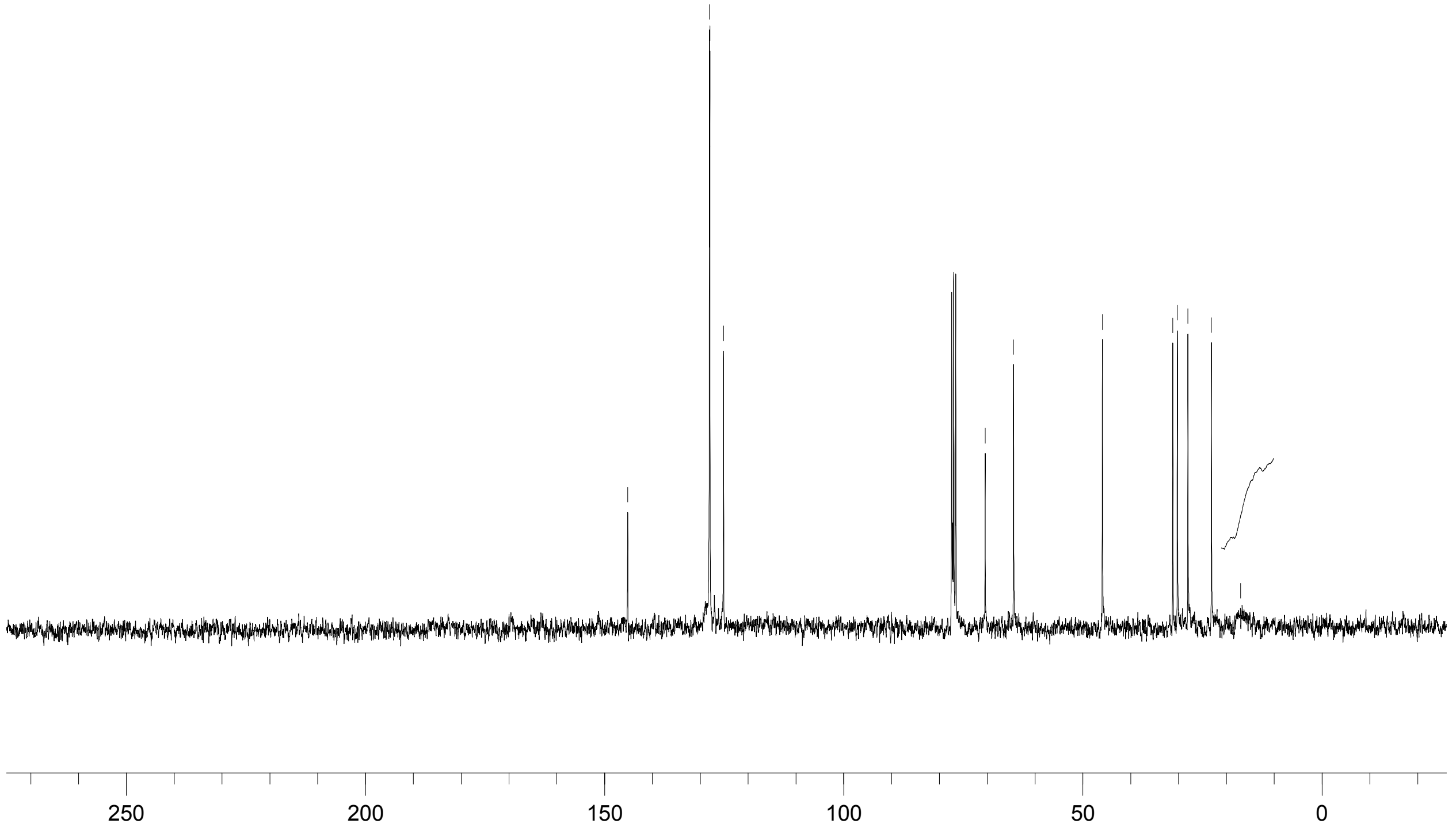

ppm (t1) 